San Jose State University

SJSU ScholarWorks

Doctoral Projects

Master's Theses and Graduate Research

Spring 4-2015

\title{
Implementation of Interprofessional Education and Physician Order Set Improves Compliance with Early Recognition and Treatment of Maternal Sepsis
}

Lori Susan Olvera

Northern California Consortium, Doctor of Nursing Practice Program, California State University, Fresno and San José State University

Follow this and additional works at: https://scholarworks.sjsu.edu/etd_doctoral

Part of the Critical Care Nursing Commons, Maternal, Child Health and Neonatal Nursing Commons, and the Nursing Midwifery Commons

\section{Recommended Citation}

Olvera, Lori Susan, "Implementation of Interprofessional Education and Physician Order Set Improves Compliance with Early Recognition and Treatment of Maternal Sepsis" (2015). Doctoral Projects. 14. DOI: https://doi.org/10.31979/etd.rzjc-dqt4

https://scholarworks.sjsu.edu/etd_doctoral/14

This Doctoral Project is brought to you for free and open access by the Master's Theses and Graduate Research at SJSU ScholarWorks. It has been accepted for inclusion in Doctoral Projects by an authorized administrator of SJSU ScholarWorks. For more information, please contact scholarworks@sjsu.edu. 


\begin{abstract}
Septic shock is rare in pregnancy; however, sepsis remains an important contributor to maternal mortality. The perinatal patient can appear deceptively well before rapidly deteriorating to septic shock. There is a need for protocols regarding early recognition and management of maternal sepsis. The Surviving Sepsis Campaign developed guidelines to provide guidance for the clinician caring for patients with severe sepsis or septic shock. The purpose of this doctoral project was to evaluate compliance with early goal directed therapy before, during, and following the implementation of a standardized physician order set and interprofessional education for nurses and physicians in the perinatal setting. The systemic inflammatory response criteria were adjusted for consideration of the physiological effects of pregnancy to accurately screen for sepsis. A retrospective study included 97 patients screening positive for sepsis from April 2014 to January 2015. The indicators for early goal-directed therapy included drawing of lactate and blood cultures, administration of $30 \mathrm{ml} / \mathrm{kg}$ crystalloid intravenous fluid bolus, additionally the administration of a broad-spectrum antibiotic to determine the effects of sepsis protocol. When comparing pre and post intervention in patients with sepsis, statistical significance was achieved for draw lactate $(\mathrm{p}=.029)$, administering a broad-spectrum antibiotic $(\mathrm{p}=.006)$, and drawing a repeat lactate $(\mathrm{p}=.034)$. In patients with severe sepsis and septic shock, statistical significance was achieved for administering a broad-spectrum antibiotic $(\mathrm{p}=.010)$. The importance of education and a perinatal sepsis protocol using a multidisciplinary approach can improve compliance with the sepsis bundles.
\end{abstract}

\title{
Lori Susan Olvera
}

April 2015 


\title{
IMPLEMENTATION OF INTERPROFESSIONAL EDUCATION AND PHYSICIAN ORDER SET IMPROVES COMPLIANCE WITH EARLY RECOGNITION AND TREATMENT OF MATERNAL SEPSIS
}

\author{
by \\ Lori Susan Olvera
}

\author{
A project \\ submitted in partial \\ fulfillment of the requirements for the degree of \\ Doctorate of Nursing Practice \\ in the School of Nursing at \\ California State University, Fresno
}

April 2015 


\section{NOTE: THIS PAGE IS OPTIONAL, AND REQUIRES PAYMENT OF AN EXTRA \$55 FEE. \\ DELETE THIS PAGE IF YOU'RE NOT REGISTERING YOUR COPYRIGHT}

(C) 2015 Lori Susan Olvera 


\section{APPROVED}

For the Department of Nursing:

We, the undersigned, certify that the Doctorate of Nursing Practice project of the following student meets the required standards of scholarship, format, and style of the university and the student's graduate degree program for the awarding of the Doctorate of Nursing Practice degree.

\section{Lori Olvera DNP-c, RN, RNC Lori Susan Olvera \\ Danette Dutra}

Danette Dutra

Nursing, CSU Fresno

Beth Stephens-Hennessy

Administration

Sutter Memorial Hospital

Laura Senn

Administration

Sutter Memorial Hospital

For the University Graduate Committee:

Dean, Division of Graduate Studies 
AUTHORIZATION FOR REPRODUCTION OF DOCTORATE OF NURSING PRACTICE PROJECT

I grant permission for the reproduction of this Doctorate of Nursing Practice project in part or in its entirety without further authorization from me, on the condition that the person or agency requesting reproduction absorbs the cost and provides proper acknowledgment of authorship.

Permission to reproduce this Doctorate of Nursing Practice project in part or in its entirety must be obtained from me.

Signature of project author: 


\section{ACKNOWLEDGMENT}

I want to acknowledge my husband, Vincent, and sons, Justin and Christopher, for supporting me through pursuit of a Doctorate of Nursing Practice degree. I couldn't have achieved academic success without the support of my loving family. I want to give special recognition to the most influential person in my life, and that is my mother, Marian Conley. My mother has been an inspiration to me both personally and professionally. She influenced me to pursue an advanced degree in nursing including BSN, MSN, and now my Doctorate of Nursing Practice degree. I want to acknowledge my Chair, Dr. Danette Dutra. Danette has been an incredible support for me while implementing a difficult, time-intensive project. Danette's positive energy and strong mentor skills have kept me motivated to complete this project. Fresno State University is fortunate to have such a kind, knowledgeable faculty member to support their students in academic achievement. Danette is absolutely an asset to their faculty team. Lastly, I want to thank my committee members, Dr. Laura Senn and Beth StephensHennessy. These women have mentored me through a very tough project. Their enthusiasm for teaching and mentoring me is beyond words. Beth StephensHennessy has taught me a lot about implementing a project and the responsibilities of advanced practice nurses. I have loved working with a wonderful team of advanced practice nurses. I want to acknowledge my Dad for being an inspiration to me to continue the work on implementing a protocol for early recognition and management of sepsis. My Dad died January 28, 2013 of Severe Sepsis. He would be here today if this protocol was in place back in 2013. Dad, you are my inspiration! 


\section{TABLE OF CONTENTS}

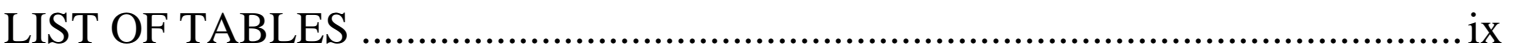

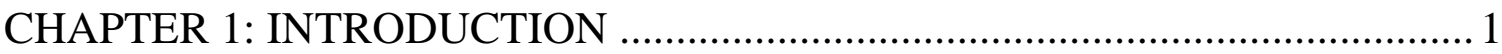

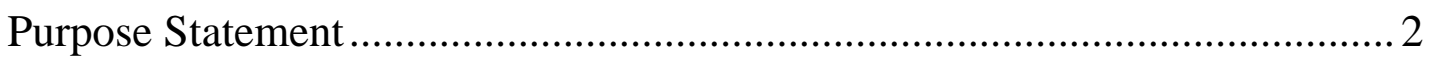

Surviving Sepsis Campaign .................................................................... 2

Surviving Sepsis Campaign and the Pregnant Woman...................................... 4

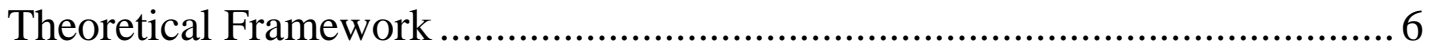

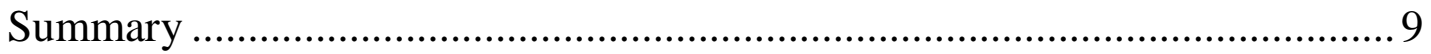

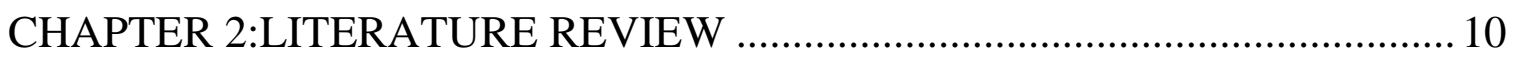

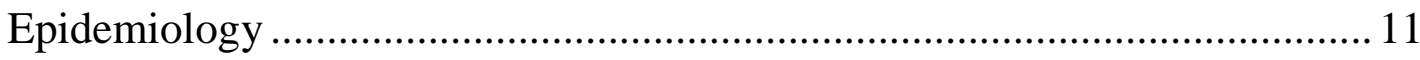

Physiologic Changes of Pregnancy .............................................................. 13

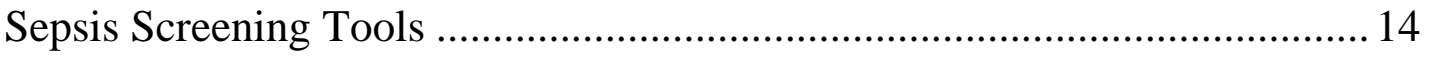

Recommendation of Protocols and Guidelines ............................................... 16

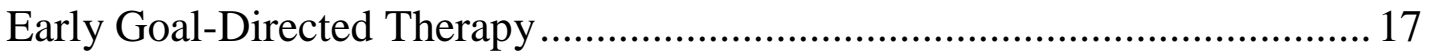

Nurse and Physician Champions.................................................................. 19

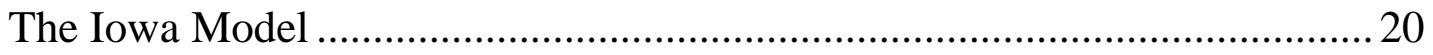

The Iowa Model in the Perinatal Setting …………....................................... 20

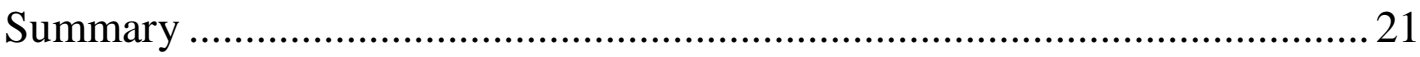

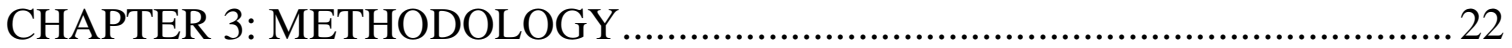

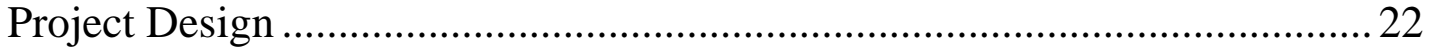

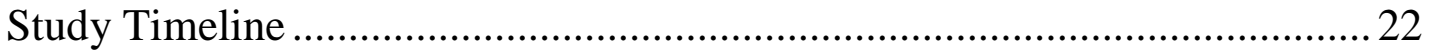

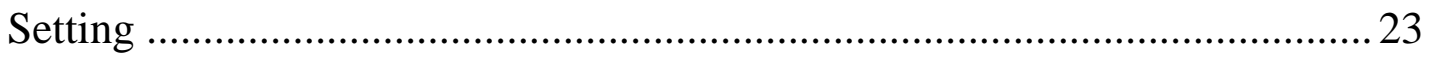

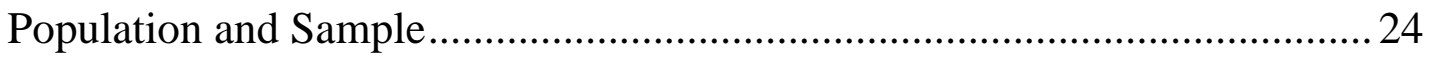

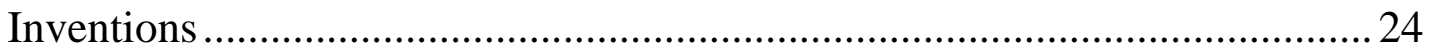




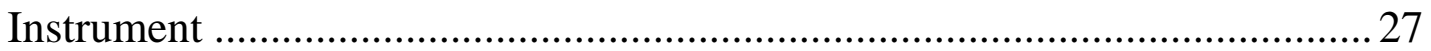

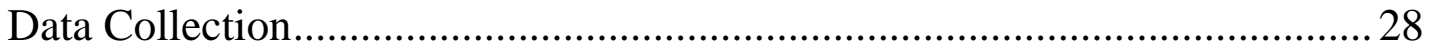

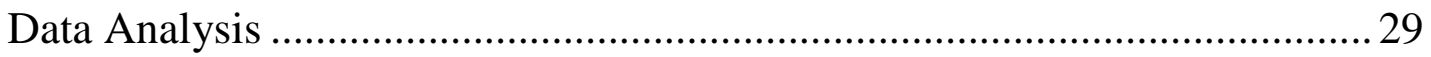

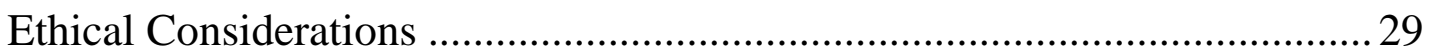

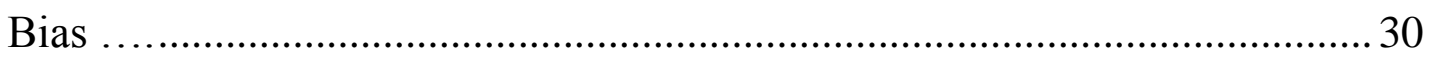

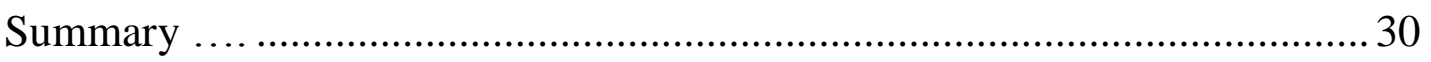

\section{CHAPTER 4: RESULTS AND DISCUSSION}

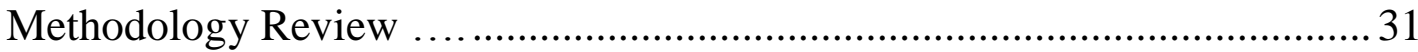

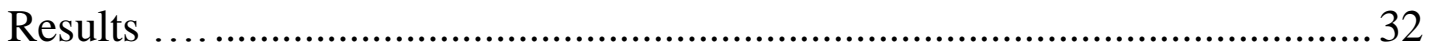

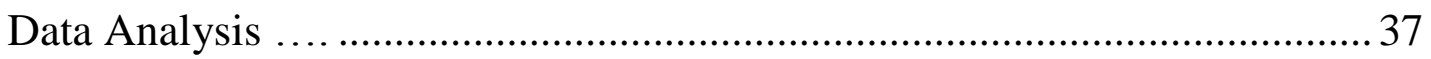

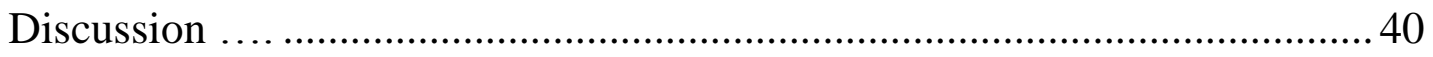

Maternal Adjusted Parameters for Sepsis Screening ................................... 40

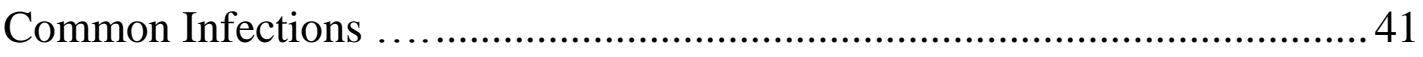

Bundle Compliance for Sepsis Patients ........................................................ 38

Bundle Compliance Indicators ....................................................................4 44

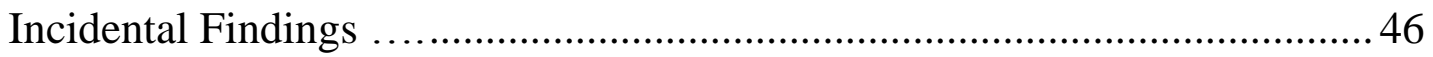

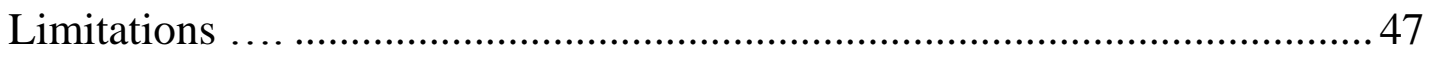

Implications for Nursing Practice .......................................................... 48

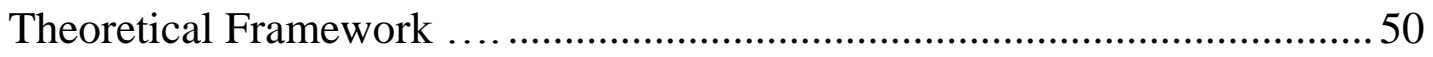

Summary … 


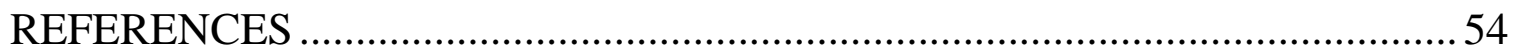

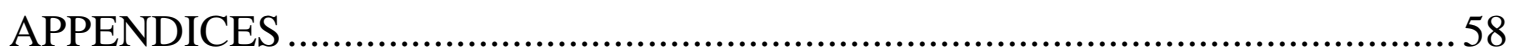

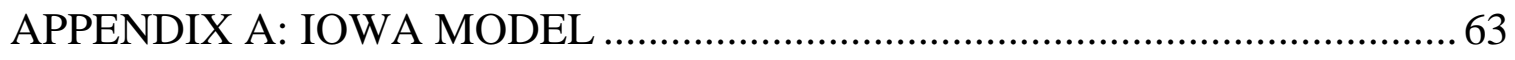

APPENDIX B: STANDARDIZED PHYSICIAN ORDDER SET ...........................65

APPENDIX C: OBSTETRICAL SEPSIS MANAGEMENT PATHWAY ............68

APPENDIX D: DATA COLLECTION TOOL ………...................................... 70 


\section{LIST OF TABLES}

Table 1. Frequency of Sepsis, Severe Sepsis, and Septic Shock from April 2014-January 2015 .

Table 2. The Number of Sepsis Patients Diagnosed with Common Infections of Pregnancy

Table 3. Bundle Compliance for Patients Meeting Sepsis Criteria Prior to Intervention 34

Table 4. Bundle Compliance for Severe Sepsis Patients Prior to Intervention..............34

Table 5. Bundle Compliance for Septic Shock Patients Prior to Intervention....... 35

Table 6. Bundle Compliance for Sepsis Patients After Intervention. ..................... 36

Table 7.Bundle Compliance for Severe Sepsis After Intervention......................... 36

Table 8. Bundle Compliance for Septic Shock Patients After Intervention............ 37

Table 9. Weighted Cross Tabulations for Lactate Drawn in Patients with Sepsis Pre and Post Intervention ................................................................ 38

Table 10. Weighted Cross Tabulations for Antibiotic Administered in Septic Patients Pre and Post Intervention. .............................................................. 38

Table 11. Weighted Cross Tabulations for Repeat Lactate Drawn in Positive Sepsis Screen Patients.

Table 12. Weighted Cross Tabulations for Broad Spectrum Antibiotic Administed in Patients with Severe Sepsis or Septic Shock.

Table 13. Percentage Rates for Bundle Compliance Indicators in Patients with Sepsis, Severe Sepsis, and Septic Shock in the Pre-Intervention Group.

Table 14. Percentage Rates for Bundle Compliance Indicators in Patients with Sepsis, Severe Sepsis, and Septic Shock in the Post-Intervention Group. 


\section{CHAPTER 1: INTRODUCTION}

Sepsis is one of the leading causes of death in the United States, and the number one cause of death in the intensive care unit (ICU) (Guinn, 2007). Sepsis, a systemic inflammatory response to an infection, is an exaggerated immune response to fight against infection, leading to dysfunction of one organ, known as severe sepsis, and eventually multiple organs known as septic shock (Dellinger, et al, 2012). Sepsis is a complex disorder that is difficult to diagnose and treat. If sepsis goes unrecognized or untreated, it can lead to severe sepsis with a mortality rate of $30-35 \%$, or septic shock with a mortality rate of 50\% (Dellinger, et al, 2012). Fortunately, septic shock is rare in pregnancy affecting $0.002-0.01 \%$ of all deliveries (Snyder, Barton, Habli, and Sibai, 2013). However, sepsis remains an important contributor to the maternal mortality. In the United Kingdom, for each maternal sepsis death, 50 women had life-threatening morbidity from sepsis (Acosta, Kurinczuk, Lucas, Tuffnell, Sellers, and Knight, 2014). There is a need for protocols for early recognition and management of maternal sepsis because the onset from systemic inflammatory response (SIRS) to severe sepsis occurs very rapidly (Acosta, Kurinczuk, Lucas, Tufnell, Sellers, and Knight, 2014). Pregnant women are more vulnerable to infection and susceptible to serious complications than non-gravid women (Joseph, Sinha, Paech, and Walters, 2009). 


\section{Purpose Statement}

The purpose of this doctoral project is to evaluate staff compliance with early goal directed therapy before and following the implementation of standardized physician order and education for nurses and physicians in the perinatal setting. The systemic inflammatory response criteria will be adjusted for consideration of the physiological effects of pregnancy to accurately screen for sepsis (See Appendix E)

\section{Surviving Sepsis Campaign}

The Surviving Sepsis Campaign (SSC), an international initiative to reduce mortality by sepsis, developed guidelines to assist practitioners in the recognition and treatment of sepsis (Nguyen, 2012). The SSC introduced bundles, a set of interventions, when grouped together, will improve patient outcomes. These set of interventions derived from evidence based practice must be implemented as a group rather than individually to achieve the best patient outcomes. Even though pregnant women were excluded from the original studies when these bundles and/or early-goal directed therapy was tested, pregnant women must be included in the general management principles for early-goal directed therapy. The resuscitation bundle or 3- hour bundle is comprised of those interventions completed within patient's arrival to the triage area or diagnosis of sepsis. These interventions are time sensitive and must be completed within 3 hours. The SIRS criteria often overlap with normal physiologic parameters during pregnancy and 
the immediate postpartum; therefore, alternative criteria must be developed to diagnose maternal sepsis (Bauer, Rajala, MacEachern, Polley, and Childen, 2014). The first care bundle is comprised of four interventions for the first 3 hours of identified sepsis (Guinn, 2011). This is called the resuscitation bundle with the goal of limiting tissue hypoxia and hypoperfusion while initiating antimicrobial therapy. The first step is to measure serum lactate to determine extent of tissue ischemia. Lactate is a marker for severe sepsis in a patient with suspected or known infection. Lactate above $2 \mathrm{mmol} / \mathrm{L}$ indicates tissue hypoxia, a sign of organ dysfunction (Dellinger, et al, 2012). Successive measurement of lactate monitors for improvement of sepsis after resuscitation measures are implemented. Secondly, blood cultures need to be collected prior to antibiotic administration. Blood cultures are important for identifying the causative pathogen, however, should not delay antibiotic administration. The third intervention is administration of broad spectrum antibiotics. When a diagnosis of severe sepsis is determined, broad-spectrum antibiotics need to be started within 3 hours for outpatient admissions and 1 hour for inpatient admissions. In the event of hypotension and/or lactate above $4 \mathrm{mmol} / \mathrm{L}$, a fourth intervention is required. The septic patient should receive $30 \mathrm{ml} / \mathrm{kg}$ of normal saline to maintain adequate blood pressure and a mean arterial pressure above 65 . 


\section{Surviving Sepsis Campaign and the Pregnant Woman}

The Surviving Sepsis Campaign (SSC) is not widely accepted in the obstetrical service line because the criteria for systemic inflammatory response (SIRS) were not defined for pregnant patients. It was difficult to apply these criteria to pregnant patients because the physiologic changes of pregnancy mimic signs of sepsis. White blood cell (WBC) counts increase throughout pregnancy (Guinn, 2011). The WBC count can be greater than 20,000 in a pregnant woman without evidence of an infection. Heart rate increases progressively during pregnancy by $10-20 \mathrm{bpm}$. This change in heart rate represents a $20 \%-25 \%$ increase over baseline (Sanghavi and Rutherford, 2014).

In pregnancy, there is a decrease in arterial pressure due to peripheral vasodilation including systolic blood pressure (SBP), diastolic blood pressure (DBP), and mean arterial pressure (MAP) (Sanghavi and Rutherford, 2014). Arterial pressures decrease to a nadir of $5-10 \mathrm{mmHg}$ below baseline with lowest drops of blood pressure at 6-8 weeks of pregnancy. The importance of comparing hemodynamic measurements with preconception values is emphasized to determine accurate assessment of changes in baseline parameters. Pregnant women typically exhibit an increase in respiratory depth while the rate remains unchanged due to stimulation of the respiratory center in the brain secondary to increased progesterone during pregnancy (Yeomans and Gilstrap, 2005). The 
respiratory rate and heart rate increases during the second stage of labor with pushing efforts, therefore, making it difficult to use the systemic inflammatory response criteria as a valid screening tool during this time (Guinn, 2011).

Changes in the respiratory physiology including an increase in tidal volume, a reduction of residual volume and functional residual capacity lead to an increase in respiratory rate and minute ventilation resulting in respiratory alkalosis with a compensatory metabolic acidosis. A pregnant woman has a diminished ability to compensate for metabolic acidosis when her condition is complicated with a severe infection (Joseph, Sinha, Paech, and Walters, 2009). Therefore, pregnant women may appear deceptively well before experiencing vascular collapse secondary to severe sepsis and/or septic shock.

The systemic inflammatory response criteria (SIRS) overlapped the normal physiological parameters during pregnancy and the immediate postpartum, therefore, SIRS criteria were adjusted to diagnose maternal sepsis. The systemic inflammatory response criteria were modified to take into account the physiological changes in pregnancy. We adopted the obstetric systemic inflammatory response criteria from a 2001 study of severe obstetric morbidity (Waterstone and Wolfe, 2001).

Common infections during pregnancy, especially if untreated, may lead to sepsis. Primary sites of infection in pregnancy are the urinary tract (pyelonephritis), the pelvic structures (chorioamnionitis and endometritis), 
surgical wounds (cesarean section), and the breasts (mastitis) (Joseph, Sinha, Paech, and Walters, 2009). Renal plasma flow and glomerular filtrate increase in pregnancy, resulting in decreased blood urea and creatinine concentrations.

During pregnancy, the smooth muscle relaxation and obstruction of the ureters results in dilatation of the renal collecting system. This leads to urinary stasis and asymptomatic bacteriuria, a precursor for pyelonephritis. Chorioamnionitis is a common obstetric complication occurring in $1-5 \%$ of term pregnancies and $25 \%$ of patients experiencing preterm delivery (Black, Hinson, and Duff, 2012).

Chorioamnionitis, a histopathologic finding of inflammation of the amnion and/or the chorion, is a result of a bacterial infection of the amniotic fluid (Fahey, 2008). The most common route of intrauterine infection is ascending bacteria from the lower genital tract. Because intraamniotic infection (IAI) and pyelonephritis are common causes of obstetric sepsis and difficult to recognize due to physiology of pregnancy, protocols and guidelines must be developed for early recognition and treatment of severe sepsis and/or septic shock. The theoretical framework identified for the project is the Iowa model.

\section{Theoretical Framework}

The Iowa model provides a theoretical framework for guiding practitioners including physicians, nurses, and advanced practice nurses in the use of evidence based practice to improve patient outcomes. The Iowa model provides a framework for decision-making and algorithm to organize the step-by-step process 
of a change project (Melynk \& Fineout-Overholt, 2011). Each step involves an activity or decision point starting with the identification of a problem to the final step of disseminating the results of the new practice.

The Iowa model begins with identifying the problem triggers or the clinical problem that needs to be changed as well as knowledge triggers, evidence-based practice, new guidelines, and institutional standards that provide support for change of the identified problem. Knowledge and problem-focused triggers assist staff to question current practice and whether patient outcomes could be improved though the use of current research findings (Titler, 2010). According to the literature, sepsis contributes to maternal deaths worldwide (Bamfo, 2013). The use of early goal-directed therapy has been proven to decrease mortality for patients presenting with severe sepsis and septic shock (Rivers, 2001; Guinn, 2011; Guinn, 2007). Patient outcomes and survivability for patients with severe sepsis and septic shock in pregnancy are improved with early detection, prompt recognition of the source of infection and targeted therapy (Barton \& Sibai, 2012). Identification of a problem is crucial for commitment of the team and healthcare professionals to be willing to change current practice. Problem triggers identified were delay of diagnosis of sepsis by the obstetrical provider or clinician, resulting in the deterioration of patient condition to severe sepsis and septic shock. Patients may appear deceptively well before deteriorating with development of septic shock, multiple organ failure, and/or death (Barton \& Sibai, 2012). There 
was lack of consistency amongst providers to follow recommended interventions to recognize and treat sepsis. Implementing a physician order set and education for early recognition and management of maternal sepsis was aligned with the organizational goals as our hospital was currently implementing early goaldirected therapy in the adult service line to decrease morbidity and mortality. The Chief of Obstetrics and Gynecology supported this change activity because the focus of the process improvement activity, direction, and outcomes were aligned with the goals of the organization.

Knowledge triggers included recommendations by researchers to implement protocols for early-goal directed therapy to treat maternal sepsis because the disease progression is more rapid as compared to non-pregnant state (Royal College of Obstetricians and Gynaecologists, 2012). After identifying triggers to improve patient outcomes, a committee was formed to critique and synthesize the literature to determine if a change in practice was warranted (Titler, 2010). Clinical nurse specialists, obstetricians, and nurse champions critiqued the literature and determined that implementation of a protocol and physician order set supporting early recognition and treatment of maternal sepsis was needed to improve patient outcomes. This change project would be piloted at a tertiary care hospital. Trialing this practice change is vital to identifying issues before instituting a regional rollout (Ciliska, DiCenso, Melynk, Fineout-Overholt, Stetler, Cullen, Larrabee, Schultz, Rycroft-Malone, Newhouse, and Dang, 2011). 
The Iowa model is desirable for multidisciplinary healthcare teams to apply evidence in practice requiring multiple steps to align clinician behavior and system support for delivery of evidence based practice (Titler, 2010). The Iowa model provides feedback loops, reflecting analysis, evaluation, and modification of change process (Ciliska, et al, 2011). This allows individualizing the evidence to the healthcare setting using a multidisciplinary team approach.

\section{Summary}

Sepsis is one of the leading causes of morbidity and mortality in the United States. Early recognition and prompt, aggressive therapy is crucial to reduce morbidity and mortality in women with suspected sepsis. Recognition of sepsis is difficult in the pregnant patient as the physiology of pregnancy mimics the systemic inflammatory response of sepsis. During labor and delivery, heart rate, white blood cell counts, and respiratory rates are high, further making it difficult to screen for sepsis. The Surviving Sepsis Campaign developed the sepsis bundles for early resuscitation and management of septic patients. A sepsis screening tool was developed to include the unique physiology of pregnancy before implementing the bundles. Patient outcomes for patients with severe sepsis and septic shock are improved with early detection, prompt recognition of the infection, and targeted therapy. The Iowa model provides a framework for guiding multidisciplinary teams in the implementation of evidence-based practice. 
CHAPTER 2: REVIEW OF LITERATURE

The purpose of this doctoral project is to evaluate staff compliance with early goal-directed therapy before and following the implementation of a standardized physician order and education for nurses and physicians in the perinatal setting. The systemic inflammatory response criteria will be adjusted for consideration of the physiological effects of pregnancy to accurately screen for sepsis.

The Surviving Sepsis Campaign (2012) guidelines for severe sepsis and septic shock recommend early recognition and initiation of evidence-based treatment to improve patient outcomes. The original studies supporting the need for early-goal directed therapy excluded pregnant women due to possible harm to fetus. As a result, the adoption of guidelines in the obstetrical service line was slow. Pregnant women are young and healthy with few comorbidities, therefore, were not included in the rollout of early-goal directed therapy for the adult population in hospitals (Guinn, 2011). Pregnant patients generally responded to common broad-spectrum antibiotics after developing an infection. In addition, the majority of pelvic infections responded well to medical or surgical intervention. These factors lead to a lower mortality rate; therefore, the benefit of early-goal directed therapy for pregnant patients was minimized.

The literature will be reviewed according to epidemiology, physiology of pregnancy, the adoption of screening tools for maternal sepsis, the importance of 
early goal-directed therapy, and recommendation of protocols and guidelines to manage maternal sepsis.

\section{Epidemiology}

Maternal sepsis accounts for $15 \%$ of maternal deaths worldwide (Bamfo, 2013). Health disparities still exist with three times higher maternal deaths reported in low-income countries as compared to high-income countries.

Obstetric patients with sepsis tend to be a younger, healthier population; therefore, morality rates compared to non-pregnant persons tend to be lower. However, the mortality rates due to severe sepsis and septic shock are increasing and most common cause of direct maternal death (Barton \& Sibai, 2012). Most importantly, these maternal deaths due to sepsis are preventable. The lack of recognition of signs of sepsis and guidelines to manage these patients contribute to these preventable deaths. A study by Kramer, et al (2009) performed a nationwide confidential inquiry into maternal mortality from 1993-2006 in the Netherlands. This study concluded that mortality from sepsis was $7.7 \%$. The cause for mortality in 38\% of the cases identified a delay in diagnosis and/or treatment. Group A streptococcal infection was is cause of morbidity in $31.8 \%$ of the cases from 2004-2006.

A retrospective study by Acosta, et al (2013) investigated the incidence and risk factors associated with uncomplicated maternal sepsis in California from 2003-2005 ( $\mathrm{N}=1598)$. The rate of severe sepsis was twice the national estimate. 
Sociodemographic disparities were noted with those who developed sepsis. Those women who progressed from sepsis to severe sepsis had known clinical risk factors, no insurance, racial or ethnic minority status, diabetes, low hospital birth volume, or chronic hypertension. Results indicated that 10 per 10,000 live births (95\% CI=9.4-10.3) developed sepsis. Women progressing to severe sepsis were 4.9 per 10,000 live births $(95 \% \mathrm{CI}=4.5-5.2)$. Preeclampsia and postpartum hemorrhage were associated with progression to severe sepsis. For every cumulative risk factor, the risk increased by $25 \%(95 \% \mathrm{CI}=10.4-32.3)$ and progression to severe sepsis increased by $57 \%(95 \% \mathrm{CI}=40.8-74.4)$.

The increase in the number of sepsis related cases are attributed to the change in demographics of women who are pregnant. More women over the age of 40 are becoming pregnant. Disorders of pregnancy such as preeclampsia, placental abruption, amniotic fluid embolism, and postpartum hemorrhage can lead to organ failure while presenting concurrently with sepsis, further contributing to maternal mortality (Sung, George, and Porter, 2011). With the increasing rates of obesity, diabetes, and cesarean delivery, the risk of infectious morbidity and mortality will increase (Guinn, 2011). Women having cesarean sections are three times more likely to develop sepsis. 


\section{Physiologic changes of Pregnancy}

The physiologic changes of pregnancy mimic the signs of sepsis making it difficult to recognize and diagnose sepsis in the obstetrical patient. The cardiovascular changes include increased heart rate, peripheral dilation leading to a decrease in blood pressure (Guinn, 2011). These physiologic changes mask the initial presentation of sepsis as well as intensify the decreased organ dysfunction seen in the septic patient. Labor and delivery is a time when physiological changes further complicate the clinical picture for the patient at risk for developing sepsis. The laboring women's heart rate and respiratory rate increase due to physical exertion. Cardiovascular changes include increases in heart rate and peripheral vasodilation leading to decrease in blood pressure (Yeomans and Gilstrap, 2005). The respiratory depth increases while the rate remains unchanged due to stimulation of the respiratory center in the brain secondary to progesterone (Yeomans and Gilstrap, 2005). The respiratory rate increases transiently during the secondary stage of labor with pushing efforts (Guinn, 2011). Cardiac output increases 50\% during delivery of fetus with large fluid shifts and significant blood loss (Guinn, 2011). Common interventions during labor \& delivery such as administration of intravenous fluids and the use of epidural anesthesia blur the clinical picture for early recognition of sepsis. Increased maternal temperature in labor may be attributed to epidural anesthesia and delay diagnosis of sepsis in the obstetrical patient. Gonen, Korobochka, Degani, and Gaitini (2000) found that 
$11.8 \%$ of women who received epidural anesthesia $(\mathrm{N}=406)$ developed an intapartum fever $\geq 37.8$ degrees Celsius. White blood cell counts during pregnancy are elevated and can increase in labor to $20,000 \mathrm{~K}$ without evidence of infection (Guinn, 2011).

Physiological changes in a pregnant woman's immune system allow tolerance to paternally derived fetal antigens (Lapinsky, 2013). The maternal immune response in pregnancy is deceased to protect the immunologically distinct fetus. There are decreased T-helper cells that play an important role in the immune response to pathogens (Acosta and Knight, 2013). As a result, the pregnant woman has a predisposition for developing severe bacterial, fungal, or viral infections. A woman having a cesarean section is at a higher risk of developing infection at the surgical site and endometrium. In addition, the pregnant woman is susceptible for developing acute respiratory distress syndrome secondary to a suppressed immune system.

\section{Sepsis Screening Tools}

The SSC guidelines recommended routine screening for sepsis to increase early identification of sepsis and implementation of early sepsis therapy (Dellinger, et al, 2012). Early identification of sepsis and implementation of evidence-based therapies will improve patient outcomes and decrease mortality. Lack of early recognition of sepsis was a major obstacle to sepsis bundle initiation. 
Sepsis screening tools were developed to identify possible septic patients to help improve patient outcomes.

Sepsis screening tools are used to evaluate for systemic inflammatory response criteria including altered body temperature, elevated pulse rate and respiratory rate and abnormal white blood cell count in the adult population. Commonly used prognostic tools used, the Apache II and III, tend to overestimate maternal mortality (Honiden, S., Abdel-Razeq, S., and Siegel, M, 2011). The physiological variables produced higher severity of illness scores because these scoring tools were not developed for pregnant women. These variables such as respiratory alkalosis, increased heart rate, increased respiratory rate, lower blood pressure, or lower hematocrit were normal variations of pregnancy.

The systemic inflammatory response criteria were developed for the nonpregnant population. SIRS criteria were not developed with maternal specific parameters for sepsis in the pregnant population. In a study by Waterstone, Bewley, and Wolfe (2001), the standard definition of sepsis included modified SIRS criteria incorporating the physiological changes in pregnancy. This definition was used to estimate incidence and predictors of severe obstetric morbidity. This was the first study to adjust the SIRS criteria for the pregnant patient and develop reproducible definitions defining severe sepsis. The adjusted SIRS criteria for perinatal patients were replicated in a study by Acosta, et al (2014). 
Bauer's et al (2013) study, conducted from 1998-2008, discovered an increase in frequency of severe sepsis from 1:15,385 (95\% CI, 1:12,987-1:18,519) to 1:7246 (95\% CI, 6329-8333). Sepsis occurred in the absence of identifiable risk factors, indicating a need for a screening program that increases sensitivity for disease detection across the entire population. This study corresponded with the study by Rivers et al (2001) demonstrating the importance of early goal-directed therapy.

\section{Recommendation of Protocols \& Guidelines}

Bauer, Bateman, Bauer, Shanks, and Mhyre (2013) collected data from the patients admitted to hospitals in a nationwide inpatient sample $(\mathrm{N}=44,999,260)$ from 1998-2008 using a stratified sampling technique. Researchers concluded that maternal sepsis was increasing in the United States. The risk of severe sepsis and sepsis related deaths increased during the study period $(\mathrm{p}<0.001)$ and $(\mathrm{p}=0.02)$. The researchers recommended for hospitals to develop system wide protocols for recognition of severe sepsis. This study period coincided with the initiation of the Surviving Sepsis Campaign and the implementation of early-goal directed therapy.

Pregnant women are susceptible to infection and more likely to develop complications (Joseph, Sinha, Paech, and Walters, 2009). According to the Centre for Maternal Child Enquiries (2011), the maternal mortality rate from sepsis is 1.13 per 100,000 maternities in 2006-2008 (95\% CI, 0.77-1.67). In 2007, the Centre for Maternal Child Enquiries (2011) identified sepsis as the leading cause 
of direct maternal deaths while overall maternal mortality decreased. Deaths were from community-acquired Group A streptococcal disease. In an effort to address the opportunity to improve patient outcomes, 10 key recommendations for improving child health and reducing maternal mortality were identified (Centre for Maternal and Child Enquiries, 2011). One of the recommendations stressed the importance of early recognition and management of sepsis in pregnant women. In addition, the routine use of an early warning system and development of guidelines and protocol for practitioners caring for obstetrical patients were recommended to improve patient outcomes. All healthcare professionals caring for pregnant or recently delivered women must have regular training and education in the early recognition of sepsis. Signs and symptoms of severe sepsis and the need for prompt assessment and treatment to prevent the rapid progression of sepsis to septic shock must be included in education of health professionals caring for obstetric women (Centre for Maternal and Child Enquiries, 2011).

\section{Early Goal-Directed Therapy}

In 2004, the Surviving Sepsis Campaign was launched to provide standardized evidence-based recommendations for early recognition and management of severe sepsis, including the resuscitation and management bundles for early goal-directed therapy (Acosta and Knight, 2013). These guidelines were revised in 2008 and 2012 (Dellinger, et al, 2012). These guidelines were intended to provide guidance for the clinician caring for a patient with severe sepsis and to 
improve patient outcomes. The first care bundle is comprised of interventions for the first 3 hours of identified severe sepsis (Guinn, 2011). This is called the resuscitation bundle with the goal of limiting tissue hypoxia and hypoperfusion while initiating antimicrobial therapy. The resuscitation care bundle included measurement of lactate level, obtaining blood cultures, administering broadspectrum antibiotics, and administering 30-ml/kg crystalloid for hypotension for hypotension or lactate $\geq 4 \mathrm{mmol} / \mathrm{L}$.

The first step is to measure serum lactate to determine extent of tissue ischemia and monitor for improvement with successive measurement of lactate levels. Lactate is a marker for severe sepsis in a patient with suspected or known infection. Lactate above $2 \mathrm{mmol} / \mathrm{L}$ indicates tissue hypoxia, a sign of organ dysfunction (Morgan and Roberts, 2013). Secondly, blood cultures need to be collected prior to antibiotic administration. Blood cultures are important for identifying the causative pathogen, however, should not delay antibiotic administration. From the time of presentation, broad -spectrum antibiotics need to be administered within 3 hours in the outpatient setting and 1 hour for inpatient admissions. In the event of hypotension and/or lactate above $4 \mathrm{mmol} / \mathrm{L}$, the patient should receive $30 \mathrm{ml} / \mathrm{kg}$ of normal saline to maintain adequate blood pressure and a mean arterial pressure above 65. For continued hypotension, vasopressor medications can be given if blood pressure is not above 90 systolic after initial fluid resuscitation. In addition, a central venous pressure catheter must be 
inserted to measure central venous pressure for accurate measurement of hemodynamic status. A urinary catheter must be inserted to measure hourly urine output to monitor renal perfusion and signs of renal failure. In addition, overall assessment of fluid balance to guide fluid therapy and prevent fluid overload is crucial. The SSC recommends hospitals to develop customized sepsis protocols using these care bundles to achieve the $25 \%$ reduction of mortality.

\section{Nurse and Physician Champions}

Physician and nurse champion must be recruited to support the project. According to Porter-O'Grady and Malloch (2011), it is important to include the opinions, values, and perspectives of frontline staff in decisions affecting their workflow. The RN champions can help disseminate information out to staff and help mentor staff with the new process. According to Porter-O'Grady and Malloch, 2011), creating opportunity for small successes to occur early and often during the implementation of innovation will motivate those individuals most affected by the change. People will not be motivated unless they feel their efforts will make a difference. Having champions that oppose the project as well as support the project can be vital to the success of the project. Having champions expressed the opposing point of view incorporates new ideas, overcome objections, and possibly convert opponents into supporters of the change project (Porter-O’Grady and Malloch, 2011). 


\section{The Iowa Model}

The Iowa Model was developed to enhance cost effectiveness, improve patient outcomes, and improve quality of care (Taylor-Pilliae, 1999). The Iowa Model proves a framework for decision-making and an algorithm to organize the step-by-step process of a change project (Melnyk \& Fineout-Overholt, 2011). This model was created to help nurses or clinicians create change through implementation of evidence base practice in the clinical setting. The model is a systematic process of implementing change through a progressive series of steps. Each step involves an activity or decision point starting with the identification of a problem to the final step of disseminating the results of the new practice. This final step is crucial for ongoing commitment by nurses to the change in practice. This model directs the clinician though the process of implementing evidencebased practice (Melynk \& Fineout-Overholdt, 2011).

\section{The Iowa Model in the Perinatal Setting}

The Iowa Model provides a framework for an entire healthcare setting, and introduces current research for implementing change. Changes in one department impacts other departments. When implementing new guidelines and staff education for recognition and management of maternal sepsis, stakeholders from all departments caring for perinatal patients must be invited to take part in the process improvement activity. The Iowa Model is useful for implementing evidence-based practice at an organizational level. The process for implementing 
guidelines to recognize and treat maternal sepsis will impact several departments, therefore the process for change needs to be defined and logical.

\section{Summary}

Sepsis is one of the leading causes of death in the United States. The SSC developed guidelines to assist practitioners in the recognition and treatment of maternal sepsis. Initially, SIRS criteria were not defined for the pregnant patient, therefore, were adjusted to include maternal parameters to assist in early identification of maternal sepsis. The primary responsibility of the nurse caring for a septic pregnant patient must be early recognition of sepsis and prompt, aggressive therapy (Guinn, 2011). Pregnant woman must be included in the general management principles for early goal-directed therapy. The resuscitation bundle or 3-hour bundle is comprised of time-sensitive interventions that must be completed in 3 hours for patients screening positive for sepsis. This set of interventions must be implemented as a group rather than individually to achieve the best patient outcomes. The use of nurse and physician champions are important when implementing guidelines and protocols for management and treatment of maternal sepsis. 


\section{CHAPTER 3: METHODOLOGY}

The goal of this project is to determine if the implementation of standardized physician orders and interprofessional education for nurses and physicians would result in an increased sepsis bundle compliance. This in turn, should enhance patient outcomes since compliance to the care bundles has been well documented in the literature to improve patient outcomes, decreased ICU admission and length of hospital stay (Dellinger, et al., 2012).

\section{Project Design}

The study design was a quality improvement (QI) activity using bundle compliance data obtained from retrospective chart review as the outcome. Staff compliance to the care bundles was measured before, during, and following the implementation of a perinatal sepsis physician order set and education for obstetrical nurses, rapid response team (RRT) nurses, and obstetricians.

\section{Study Timeline}

- A multidisciplinary team including pharmacy, ICU staff, perinatal staff, and clinical nurse specialists was formed to implement project. Physician and nurse champions were recruited to support the project. A rollout date was determined as well as the establishment of regular monthly meetings to examine the process. 
- Education was provided to physicians and nurses from August through November 2014.

- A new perinatal sepsis physician order set was implemented in October 2014.

- Every month, the RRT submitted a list of obstetrical patients identified as sepsis screen positive to principle investigator

- Through medical chart review, the indicators for early-goal directed therapy were studied retrospectively including serum lactate, blood cultures, administration of intravenous fluid bolus, and administration of broadspectrum antibiotic within 1 hour.

- Data was abstracted monthly to follow progress, while sharing data with stakeholders every month following the implementation of the protocol to determine next steps and provide motivation for continued improvement. Recommendations from stakeholders helped focus interventions or revisions to existing process.

\section{Setting}

This quality improvement activity was conducted at a large community center in Northern California. This hospital is part of an integrated health care system, and provides Level 3 Maternity services and Level 4 Neonatal services with approximately 5500 births per year. There are approximately 350 registered nurses and 50 physicians employed in the perinatal service line. Designated as a 
regional referral center, the hospital provides high-risk maternity services and receives neonatal and maternal transports from other hospitals in Northern California.

\section{Population and Sample}

The population consisted of all nurses and physicians who work in Labor \& Delivery, High Risk Maternity, and Maternal Newborn units. The sample consisted of the staff who actually cared for the obstetrical patients who screened positive for sepsis.

Using a retrospective chart review, staff compliance to the indicators of early goal-directed therapy was measured before and following implementation of a perinatal sepsis physician order set and education for nurses and physicians. Inclusion criteria were patients screening positive for sepsis between April 2014 and January 2015. Exclusion criteria was patients less than 20 weeks pregnant or patients not meeting sepsis definition criteria. There were 99 charts reviewed in which 97 patients met criteria for study.

\section{Interventions}

Two physician champions were recruited to support the project and provide education to other physicians within the service line. The importance of the champion at an organizational level was an asset for initiating change, motivating others to adopt the change, and sustaining the change (Shaw, 2012). Physician "buy in" was crucial for the success of the project. Poster board presentation of 
sepsis education and evidence-based literature were placed in the physician break room for review.

Education for physicians was implemented first as physician support and acquisition of knowledge regarding maternal sepsis was important to avoid resistance of the new protocol. Education was provided for obstetrical nurses and the rapid response team nurses on numerous occasions from August through November 2014. RN champions were recruited to educate every nurse 1:1 regarding the new physician order set. The focus of education included review of sepsis screening, standardized physician order set and evidence-based practice for the recognition and management of the obstetrical patient with severe sepsis and/or septic shock. Poster presentation, case studies, and evidence-based literature were displayed for nurses to review. A single sheet, quick reference guides was provided to all staff (See Appendix C). Formal two-hour educational sessions were provided to increase awareness for the importance of early recognition and management of maternal sepsis.

A computer based education program was developed for all nurses to test their knowledge regarding maternal sepsis and the application of the care bundles for early recognition and management of maternal sepsis. The nurse did not have to complete module training if the pre-test score was above $80 \%$. Completion of the computer based learning module and a post-test was mandatory for those nurses who did not pass the pre-test. 
The physician order set support the bundles of care recommended by the Surviving Sepsis Campaign (2012). The physician orders guide the obstetrician and nurse in the application of evidence-based practice. The physician orders included interventions that must be completed by the practitioner while other interventions were recommendations to provide point of care decisionmaking capability. See Appendix B for sample of physician order set.

Pharmacists were important stakeholders and champions for rollout of this quality and safety initiative. Antimicrobial stewardships were included on the team who had strong knowledge of clinical microbiology, pharmacology, and antimicrobial agents to treat infections. The addition of antimicrobial stewardships improves patient care, reduce rates of resistance and prolong the longevity of the limited number of antimicrobial agents available to treat infections. The physicians and pharmacists are important stakeholders in the successful rollout of this project. According to Kirschner, et al (2012), including stakeholders who will be affected by the process improvement activity is key to the successful implementation of this quality initiative.

Engaging frontline leaders in the implementation of the project was crucial to its success. Perinatal Clinical Nurse Specialists (CNS) and perinatal management team were important stakeholders and mentors for the successful rollout of this project. These stakeholders were crucial to aligning the goals of the project with the goals of the organization. It was important to ensure the goals of 
innovation advance the interest of the organization (Porter-O'Grady and Malloch, 2011).

\section{Instrument}

A data collection tool, composed by author, was used to collect and measure data on compliance indicators (Appendix D). Content validity of the data collection tool was established because a team of experts reviewed it. The tool measured number of patients screening positive for sepsis as well as number of patients who progressed to severe sepsis and septic shock. Diagnosis of chorioamnionitis, pyelonephritis, and urinary tract infections were measured because common infections can lead to sepsis. Compliance indicators were measured including:

1) Lactate is measured within 3 hours of diagnosis of sepsis

2) Blood cultures performed prior to the initiation of antibiotics

3) Antibiotics given within 1 hour of diagnosis of sepsis

4) Broad-spectrum antibiotics given within 1 hour of suspected severe sepsis in the inpatient setting and within 3 hours in the outpatient setting

5) Intravenous crystalloid fluids $30 \mathrm{ml} / \mathrm{kg}$ given immediately upon diagnosis of severe sepsis or septic shock

6) Repeat measurement of lactate.

Using the correct terminology in physician history and physicals was important for proper coding. For educational purposes, this researcher monitored 
for agreement between physician notes and use of perinatal sepsis physician orders. When the perinatal sepsis orders were used, the physician notes should reflect the physician's critical thinking related to a possible sepsis diagnosis.

\section{Data Collection}

All medical records for perinatal patients with positive sepsis screens in the perinatal setting were included in the study from April 2014-January 2015. Patients with a new infection with presence of 2 SIRS criteria were the point of enrollment for medical chart review. The elements in the resuscitation bundle as described in the SSC bundles of care were measured. Medical charts of patients identified with sepsis were reviewed for adherence to the sepsis bundles according to the Surviving Sepsis Campaign (Surviving Sepsis Campaign, 2012). As early goal-directed therapy is time sensitive, it was important to audit the time a diagnosis of sepsis is made. The outcome measure was compliance with the bundle elements with an emphasis on strict timing. Delay in interventions could be detrimental to patient outcomes. Measurement of the timing of lactate, blood cultures, initiation of broad spectrum antibiotic, and fluid resuscitation determined health professional's adherence to care bundles. Repeat lactate levels were measured to ensure that lactate was decreasing after the administration of fluids. The use of frequency tables displaying results in percentages was evaluated over time. Measurements of these interventions determined compliance with the care bundles as recommended by SSC. 


\section{Data Analysis}

Frequency tables were completed with data from the medical record audits and displayed percentages of compliance for monthly results. Using descriptive statistics such as frequency and percentage tables allow health care professionals to follow the department's compliance with bundles. Improvement or regressions were noted over time. Monthly analysis was completed with dissemination of the reports to physicians and staff. Analysis of the reports drove interventions for further education and process improvement activities. The team looked for patterns and implemented creative plans to resolve problematic issues in process. See Appendix D for list of components measured.

\section{Ethical Considerations}

Internal Review Board approval was obtained from the hospital in which the study was conducted and the author's university, California State University, Fresno. This researcher made every reasonable effort to limit protected health information to the minimum necessary to accomplish the intended purpose of use, disclosure or request. A list of all obstetric patients diagnosed with sepsis was given to the researcher. This list was kept in a locked office at the hospital. A subject number was assigned as an identifier, instead of a name or medical record number. After information was entered into SPSS, the list was shredded in office. 


\section{Bias}

Bias was minimal. Potential bias exists with the data collection tool. The data collection tool was not a validated or pretested instrument. Therefore, the author's biases may have inadvertently affected the creation of the data collection tool. However, the author created a data collection tool similar to the one found on the Surviving Sepsis Campaign's website.

The documentation of timing of the intravenous fluids was not always clear, therefore, the author needed to make judgments as to the approximate timing of the intravenous fluids.

\section{Summary}

The purpose of this study was to determine perinatal healthcare professional's adherence to the care bundles according to the Surviving Sepsis Campaign. Education for physicians and nurses was provided from August to November 2014. Medical charts were audited using a data collection tool for bundle compliance for patients screening positive with sepsis from April 2014 to January 2015. Data was analyzed using descriptive statistics. The use of frequency tables displaying results in percentages were evaluated over time to determine the effects of education and the implementation of a protocol for managing maternal sepsis. 
CHAPTER FOUR: RESULTS \& DISCUSSION

The purpose of this project was to determine if the implementation of standardized physician orders and interprofessional education for nurses and physicians would result in increased sepsis bundle compliance.

\section{Methodology Review}

Medical charts of patients screening positive for sepsis were reviewed from April 2014 to January 2015 to determine if implementation plan increased sepsis bundle compliance. The education began in August 2014. All data for the four month period from April to July 2014 were considered pre-intervention data. All data collected for the five month period from August 2014 to January 2015 were deemed post-intervention data. Data were collected for three parameters: sepsis criteria, severe sepsis, and septic shock. For patients meeting sepsis criteria, four bundle compliance indicators were measured. The first three bundle compliance indicators were used to measure total bundle compliance. The administration of intravenous fluid bolus was measured, however, not required to meet the bundle compliance for sepsis.

1) Draw lactate

2) Draw blood culture

3) Administer broad-spectrum antibiotic.

4) Administer intravenous fluid bolus

5) Total bundle compliance

For patients meeting severe sepsis and septic shock, bundle compliance indicators were used, plus total bundle compliance if the first five measures were met in a timely manner: 
1) Draw lactate

2) Draw blood Cultures

3) Administer $30 \mathrm{ml} / \mathrm{kg}$ crystalloid intravenous solution

4) Administer broad-spectrum antibiotic.

5) Draw repeat lactate

6) Total bundle compliance

To measure the difference in bundle compliance pre-and post-intervention, data from the first time period was compared to data from the second time period. Data from the initial infection were measured separately to determine the source of infection.

Frequency and percentage tables were used to analyze the differences. Addition, one criteria point varied due to contextual factors. For example, the timing of antibiotic was measured according to patient setting since the setting determined the time frame for when antibiotic should be given. For the element to be scored as meeting criteria in the inpatient setting, an antibiotic must be given in 1 hour, whereas, in the outpatient setting, the broad-spectrum antibiotic must be given in 3 hours. For the purpose of reporting results, regardless of the setting, the timely administration of a broad-spectrum antibiotic was collapsed into one variable.

\section{Results}

Table 1 displays the frequency of sepsis, severe sepsis, and septic shock from April 2014 to January 2015. There were 99 patients who met the sepsis criteria from April 2014 to January 2015. Ninety eight percent of the patients screening positive for sepsis by the RN ( $\mathrm{N}=99)$ were confirmed sepsis positive by the Rapid Response Team 
nurse ( $\mathrm{N}=97)$. For patients meeting the sepsis criteria, 48.5\% ( $\mathrm{N}=47)$ progressed to severe sepsis while $7.2 \%(\mathrm{~N}=7)$ progressed to septic shock.

Table 1: Frequency of Sepsis, Severe Sepsis, and Septic Shock from April 2014-January 2015

\begin{tabular}{lll}
\hline & Frequency (Yes) & Percent \\
\hline Confirmed Sepsis Positive & 97 & 98 \\
Severe Sepsis & 47 & 48.5 \\
Septic Shock & 7 & 7.2 \\
\hline
\end{tabular}

Since common infections can lead to sepsis, and the type of antibiotic used is dependent on the type of infection, it was important to look at the source of infection. Table 2 displays the number of patients diagnosed with common infections of pregnancy. For patients meeting the sepsis criteria $(\mathrm{n}=97), 46.4 \%(\mathrm{~N}=45)$ had a diagnosis of chorioamnionitis, 14.4\% ( $\mathrm{N}=14)$ had pyelonephritis, 5.2\% $(\mathrm{N}=5)$ had endometritis, and $5.2 \%(\mathrm{~N}=5)$ had a urinary tract infection. The other 29 patients who were diagnosed with sepsis, the sources of infection were undetermined.

Table 2: The Sources of Infection for Patients Diagnosed with Sepsis during Pregnancy

\begin{tabular}{lll}
\hline & Frequency & Percent \\
Chorioamnionitis & 45 & 46.4 \\
Pyelonephritis & 14 & 14.4 \\
Endometritis & 5 & 5.2 \\
Urinary Tract Infection & 5 & 5.2 \\
Unknown & 29 & 29 \\
\hline
\end{tabular}

During the pre-intervention period, there were thirty-one (31) patients meeting the sepsis criteria. Of these 31 patients, $74.2 \%(\mathrm{~N}=23)$ had their lactate drawn, $38.7 \%$ $(\mathrm{N}=12)$ had their blood culture drawn, and $77.4 \%(\mathrm{~N}=24)$ received a broad-spectrum antibiotic within the recommended time. The administration of a $30 \mathrm{ml} / \mathrm{kg}$ bolus of crystalloid intravenous solution is only recommended for patients meeting organ 
dysfunction criteria in patients with severe sepsis and/or septic shock. The IV bolus was measured, however, not required for patients meeting sepsis criteria. Table 3 displays bundle compliance for patients meeting sepsis criteria prior to intervention.

Table 3: Bundle Compliance for Patients Meeting Sepsis Criteria (N=31) Prior to Intervention

\begin{tabular}{llll}
\hline $\begin{array}{l}\text { Compliance } \\
\text { Indicators }\end{array}$ & $\begin{array}{l}\text { Frequency } \\
\text { (Yes) }\end{array}$ & $\begin{array}{l}\text { Frequency } \\
(\text { No) }\end{array}$ & $\begin{array}{l}\text { Percent } \\
(\%)\end{array}$ \\
Draw Lactate & 23 & 8 & 74 \\
Draw Blood Culture & 12 & 19 & 38.70 \\
Fluid Bolus & 20 & 11 & 64.5 \\
Administer Broad-Spectrum & 24 & 7 & 77.40 \\
Antibiotic & & & \\
Bundle Met & 12 & 19 & 38.70 \\
\hline
\end{tabular}

Table 4 displays bundle compliance for severe sepsis patients prior to intervention. During the pre-intervention period, there were thirteen (13) patients who met criteria for severe sepsis. Of these 13 patients, $100 \%(\mathrm{~N}=13)$ had their lactate drawn, 46.2\% $(\mathrm{N}=6)$ had their blood culture drawn, $76.9 \%(\mathrm{~N}=10)$ received an intravenous bolus of 30ml/kg crystalloid solution, and $76.9 \%(\mathrm{~N}=10)$ received a broad-spectrum antibiotic within recommended time. A repeat was drawn $69.2 \%(\mathrm{~N}=9)$ of the time. All components of bundle compliance were completed $53.8 \%(\mathrm{~N}=7)$ of the time in these patients.

Table 4: Bundle Compliance for Severe Sepsis Patients (N=13) Prior to Intervention

\begin{tabular}{llll}
\hline Compliance Indicator & $\begin{array}{l}\text { Frequency } \\
\text { (Yes) }\end{array}$ & $\begin{array}{l}\text { Frequency } \\
(\mathrm{No})\end{array}$ & $\begin{array}{l}\text { Percent Met } \\
(\%)\end{array}$ \\
Draw Lactate & 13 & 0 & 100 \\
Draw Blood Culture & 6 & 7 & 46.2 \\
Fluid Bolus & 10 & 3 & 76.9 \\
Administer Broad-Spectrum & 10 & 3 & 76.9 \\
Antibiotic & & & \\
Bundle Met & 7 & 6 & 53.8 \\
Draw Repeat Lactate & 9 & 4 & 69.2 \\
\hline
\end{tabular}


Table 5 displays bundle compliance for septic shock patients prior to intervention. During the pre-intervention period, three patients met the criteria for septic shock. Of these 3 patients, $100 \%(\mathrm{~N}=3)$ had a lactate drawn, $66.7 \%(\mathrm{~N}=2)$ had a blood culture drawn, received a 30ml/kg crystalloid intravenous fluid bolus while $66.7 \%(\mathrm{~N}=2)$ received a broad-spectrum antibiotic. A repeat lactate was drawn until the lactate was less than 2 every 6 hours in $66.7 \%(\mathrm{~N}=2)$ of the patients. The resuscitation bundle was met $66.7 \%(\mathrm{~N}=2)$ of the time in septic shock patients.

Table 5: Bundle Compliance for Septic Shock Patients (N=3) Prior to Intervention

$\begin{array}{lll}\begin{array}{l}\text { Frequency } \\ (\text { Yes })\end{array} & \begin{array}{l}\text { Frequency } \\ (\mathrm{No})\end{array} & \begin{array}{l}\text { Percent Met } \\ (\%)\end{array}\end{array}$

\begin{tabular}{llll} 
Draw Lactate & 3 & 0 & 100 \\
Draw Blood Culture & 2 & 1 & 66.7 \\
Fluid Bolus & 2 & 1 & 66.7 \\
Administer Broad-Spectrum & 2 & 1 & 66.7 \\
Antibiotic & & & \\
Bundle Met & 2 & 1 & 66.7 \\
Draw Repeat Lactate & 2 & 1 & 66.7 \\
\hline
\end{tabular}

Table 6 displays bundle compliance for sepsis patients after intervention. During the post-intervention period, sixty-six (66) patients met the sepsis criteria. Of these 66 patients, 90.9\% ( $\mathrm{N}=60)$ had their lactate drawn, 43.9\% (N=29) had their blood culture drawn, 95.4\% (N=63) received a broad-spectrum antibiotic within recommended time frame. 
Table 6: Bundle Compliance for Sepsis Patients ( $\mathrm{N}=66$ ) After Intervention

$\begin{array}{lll}\begin{array}{l}\text { Frequency } \\ (\text { Yes })\end{array} & \begin{array}{l}\text { Frequency } \\ (\mathrm{No})\end{array} & \begin{array}{l}\text { Percent Met } \\ (\%)\end{array}\end{array}$

\begin{tabular}{llll} 
Draw Lactate & 60 & 6 & 90.9 \\
Draw Blood Culture & 29 & 37 & 43.9 \\
Fluid Bolus & 48 & 18 & 73 \\
Administer Broad-Spectrum & 63 & 3 & 95.4 \\
Antibiotic & & & \\
Bundle Met & 37 & 29 & 45.5 \\
Draw Repeat Lactate & 52 & 14 & 79 \\
\hline
\end{tabular}

Table 7 displays bundle compliance for severe sepsis after intervention. During the post-intervention period, thirty four patients met the severe sepsis criteria. Of these 34 patients, $97.1 \%(\mathrm{~N}=33)$ had their lactate drawn, 55.9\% (N=19) had their blood culture drawn, 73.5\% $(\mathrm{N}=25)$ received an intravenous fluid bolus, 97\% $(\mathrm{N}=33)$ received a broadspectrum antibiotic, and $82.4 \%(\mathrm{~N}=28)$ had a repeat lactate drawn. The bundle for all interventions was completed $52.9 \%(\mathrm{~N}=18)$ of the time.

Table 7: Bundle Compliance for Severe Sepsis (N=34) After Intervention

\begin{tabular}{llll}
\hline & $\begin{array}{l}\text { Frequency } \\
\text { (Yes) }\end{array}$ & $\begin{array}{l}\text { Frequency } \\
(\text { No) }\end{array}$ & $\begin{array}{l}\text { Percent Met } \\
(\%)\end{array}$ \\
Draw Lactate & 33 & 1 & 97.1 \\
Draw Blood Culture & 19 & 15 & 55.9 \\
Fluid Bolus & 25 & 9 & 73.9 \\
Administer Broad-Spectrum & 33 & 1 & 97.1 \\
Antibiotic & & & \\
Bundle Met & 18 & 16 & 52.9 \\
Draw Repeat Lactate & 28 & 6 & 82.4 \\
\hline
\end{tabular}

Table 8 displays bundle compliance for septic shock patients after intervention. During the post-intervention period, four patients met the criteria for septic shock. Of these 4 patients, $75 \%(\mathrm{~N}=3)$ had a lactate drawn, $75 \%(\mathrm{~N}=3)$ had a blood culture drawn, $100 \%(\mathrm{~N}=4)$ received a 30ml/kg crystalloid fluid bolus, $100 \%(\mathrm{~N}=4)$ received a broad 
spectrum antibiotic. A repeat lactate was drawn until less $2 \mathrm{mmol} / \mathrm{L}$ every 6 hours in $100 \%(\mathrm{~N}=4)$ of the time. The bundle was met $100 \%(\mathrm{~N}=4)$ of the time for these patients.

Table 8: Bundle Compliance for Septic Shock Patients (N=4) After Intervention

$\begin{array}{lll}\begin{array}{l}\text { Frequency } \\ \text { (Yes) }\end{array} & \begin{array}{l}\text { Frequency } \\ (\mathrm{No})\end{array} & \begin{array}{l}\text { Percent Met } \\ (\%)\end{array}\end{array}$

\begin{tabular}{llll} 
Draw Lactate & 3 & 1 & 75 \\
Draw Blood & 3 & 1 & 75 \\
Culture & & 0 & 100 \\
Fluid Bolus & 4 & 0 & 100 \\
Broad Spectrum & 4 & & \\
Antibiotic & & 0 & 100 \\
Bundle Met & 4 & 0 & 100 \\
Repeat Lactate & 4 & & \\
\hline
\end{tabular}

\section{Data Analysis}

The Chi Square test was used to quantify the relationship between pre-

intervention and post-intervention bundle compliance. The purpose of this project was to determine whether the implementation of interprofessional education and a standardized physician order set made a significant difference. Assumptions of the cross tabulations were met to perform the chi square test. A $p$ value of less than 0.05 was considered significant. In order to meet the assumptions of the cross tabulations, the severe sepsis and septic shock were collapsed into one group.

Table 9 displays cross tabulations for lactate drawn in patients screening positive for sepsis pre- and post-intervention. The chi square value was 4.442 with $\mathrm{df}=1$ with $p=.029$. Lactate drawn when comparing pre and post intervention was statistically significant $(p<.05)$ 
Table 9: Weighted Cross Tabulations for Lactate Drawn in Patients with Sepsis Pre and Post Intervention

\begin{tabular}{lllc}
\hline & Lactate Drawn (No) & $\begin{array}{l}\text { Lactate Drawn } \\
\text { (Yes) }\end{array}$ & P Value \\
\hline Pre-Intervention & $8(25.8 \%)$ & $23(74.2 \%)$ & .029 \\
Post-Intervention & $6(9.1 \%)$ & $60(90.9 \%)$ & \\
\hline
\end{tabular}

Table 10 displays weighted cross tabulations for antibiotic administered in septic patients' pre and post intervention. When isolating cases for patients screening positive for sepsis who received a broad-spectrum antibiotic, pre and post intervention cases were examined using cross tabulation. The chi square value was 7.420 with $\mathrm{df}=1$ and $p=.006$. Statistical significance was achieved with $p<.01$.

Table 10: Weighted Cross Tabulations for Antibiotic Administered in Septic Patients Pre and Post Intervention

\begin{tabular}{llll}
\hline & Broad Spectrum & Broad-Spectrum & $p$ Value \\
& Antibiotic & Antibiotic & \\
& Administered (No) & Administered (Yes) & \\
Pre-Intervention & $7(22.6 \%)$ & $24(77.4 \%)$ & .006 \\
Post-Intervention & $3(4.5 \%)$ & $63(95.5 \%)$ & \\
\hline
\end{tabular}

Cross tabulations for patients screening positive for sepsis pre and post intervention were performed for drawing a repeat lactate. The value is 4.510 with $\mathrm{df}=1$ and statistically significance noted with $p=.034(\mathrm{p}<.05)$. Table 11 displays weighted cross tabulations for repeat lactate drawn in positive sepsis screen patients.

Table 11: Weighted Cross Tabulations for Repeat Lactate Drawn in Positive Sepsis Screen Patients

\begin{tabular}{llll}
\hline & Repeat Lactate & Repeat Lactate & $p$ Value \\
& Drawn (No) & Drawn (Yes) & \\
Pre-Intervention & $13(41.9 \%)$ & $18(58.1 \%)$ & .034 \\
Post-Intervention & $14(21.2 \%)$ & $52(78.8 \%)$ & \\
\hline
\end{tabular}


Table 12 displays weighted cross tabulations for broad-spectrum antibiotic administered in a patients with severe sepsis and septic shock. When isolating cases for severe sepsis and septic shock, administration of a broad-spectrum antibiotic was examined pre and post intervention using cross tabulation. The chi square value was 6.705 with $\mathrm{df}=1$ and $p=.010$. Statistical significance achieved with $\mathrm{p}<.05$.

Table 12: Weighted Cross Tabulations for Broad Spectrum Antibiotic Administered in Patients with Severe Sepsis or Septic Shock

\begin{tabular}{llll}
\hline & Broad Spectrum & Broad Spectrum & $P$ Value \\
& Antibiotic & Antibiotic & \\
& Administered (No) & Administered (Yes) & \\
Pre-Intervention & $4(25 \%)$ & $12(75 \%)$ & .010 \\
Post-Intervention & $1(2.6 \%)$ & $37(97.4 \%)$ & \\
\hline
\end{tabular}

Table 13 displays the percentage rates of bundle compliance indicators including draw lactate, blood culture, repeat lactate, administer intravenous fluid bolus and broad spectrum antibiotic for sepsis $(\mathrm{N}=31)$, severe sepsis $(\mathrm{N}=13)$, and septic shock $(\mathrm{N}=3)$ in the pre-intervention group.

Table 13: Percentage Rates for Bundle Compliance Indicators in Patients with Sepsis, Severe Sepsis, and Septic Shock in the Pre-Intervention Group

$\begin{array}{lllllll}\begin{array}{l}\text { Number } \\ (\mathrm{N})\end{array} & \begin{array}{l}\text { Draw } \\ \text { Lactate }\end{array} & \begin{array}{l}\text { Blood } \\ \text { Culture }\end{array} & \begin{array}{l}\text { Fluid } \\ \text { Bolus }\end{array} & \begin{array}{l}\text { Broad } \\ \text { Spectrum } \\ \text { ATB }\end{array} & \begin{array}{l}\text { Bundle } \\ \text { Met }\end{array} & \begin{array}{l}\text { Repeat } \\ \text { Lactate }\end{array} \\ 31 & 74 \% & 38.7 \% & 64.5 \% & 77.4 \% & 38.7 \% & 79 \% \\ 13 & 100 \% & 46.2 \% & 76.9 \% & 76.9 \% & 53.8 \% & 69.2 \% \\ 3 & 100 \% & 66.7 \% & 66.7 \% & 66.7 \% & 66.7 \% & 66.7 \%\end{array}$

Table 14 displays the percentage rates for bundle compliance indicators including draw lactate, blood culture, repeat lactate, and administer intravenous fluid bolus and broad-spectrum antibiotic in patients with sepsis, severe sepsis, and septic shock. 
Table 14: Percentage Rates for Bundle Compliance Indicators in Patients with Sepsis, Severe Sepsis, and Septic Shock in the Post-Intervention Group

\begin{tabular}{llllllll}
\hline & $\begin{array}{l}\text { Number } \\
(\mathrm{N})\end{array}$ & $\begin{array}{l}\text { Draw } \\
\text { Lactate }\end{array}$ & $\begin{array}{l}\text { Blood } \\
\text { Culture }\end{array}$ & $\begin{array}{l}\text { Fluid } \\
\text { Bolus }\end{array}$ & $\begin{array}{l}\text { Broad- } \\
\text { Spectrum }\end{array}$ & $\begin{array}{l}\text { Bundle } \\
\text { MTB }\end{array}$ & $\begin{array}{l}\text { Repeat } \\
\text { Lactate }\end{array}$ \\
$\begin{array}{l}\text { Sepsis } \\
\text { Severe }\end{array}$ & 66 & $90.9 \%$ & $43.9 \%$ & $73 \%$ & $95.4 \%$ & $45.5 \%$ & $79 \%$ \\
$\begin{array}{l}\text { Sepsis } \\
\text { Septic } \\
\text { Shock }\end{array}$ & 4 & $97.1 \%$ & $55.9 \%$ & $73.5 \%$ & $97.1 \%$ & $52.9 \%$ & $82.4 \%$ \\
\hline
\end{tabular}

\section{Discussion}

The results of this study show that the overall effects of the interventions significantly increase bundle compliance in three of the criteria, and remained the same or increased slightly for the other criteria. A discussion of the findings will be examined including sepsis screening using adjusted maternal parameters for pregnancy, common infections, bundle compliance indicators, and comparison of bundle compliance between patients with sepsis and severe sepsis and septic shock in pre and post intervention groups.

\section{Maternal Adjusted Parameters for Sepsis Screening}

To accurately screen for sepsis in the obstetrical patient, the sepsis screening tool criteria was adjusted for the pregnant woman. The systemic inflammatory response criteria can be a sign of changing biochemistry associated with labor and delivery, a normal physiological finding in the pregnant woman, as well as a clinical sign of a severe infection. To screen for maternal sepsis, the maternal heart rate was adjusted to $100 \mathrm{BPM}$ and the white blood cell was adjusted to $20,000 \mathrm{dl} / \mathrm{ml}$ to account for the physiologic changes in the pregnant and postpartum patient (Stephens-Hennessy, 2012). 
If a patient screens positive for sepsis, the rapid response nurse is called to the bedside to evaluate the patient. There were 99 patients screening positive for sepsis, whereas the RRT nurse confirmed that 97 patients met the sepsis criteria. There was a $98 \%$ occurrence rate for nurses to accurately screen for sepsis in perinatal patients. Those patients screening positive for sepsis either progressed or initially had signs of acute organ dysfunction, indicating that $48.5 \%$ of the patients had severe sepsis. A similar finding by Martin, et al (2003) found that $34 \%$ of septic adult patients had severe sepsis.

\section{Common Infections}

The most common infection was chorioamnionitis (46.4\%) followed by pyelonephritis (14.4\%). These findings are consistent with the literature stating that maternal sepsis is primarily the result of a pelvic infection due to chorioamnionitis, endometritis, urinary tract infections, and wound infections (Guinn, Abel \& Tomlinson, 2007; Bauer, et al, 2013).

\section{Bundle Compliance}

Total bundle compliance was higher in all of the post intervention groups, except for the severe sepsis groups, where the pre- was almost identical to the post-intervention group's findings (53.8\% and 52.9\% respectively). See Table 15.

Bundle compliance indicators were reviewed for significance in pre- and postintervention groups. Bundle compliance indicators reaching statistical significance when comparing pre- and post-intervention group included draw lactate, repeat lactate, and administer broad-spectrum antibiotic(s). 
The percentage rates for the four criteria ranged from $38-74 \%$ for the sepsis only pre-intervention group. The timely administration of the appropriate broad-spectrum antibiotic ranged from $67-77 \%$ for the three levels of sepsis in the pre-intervention group. In comparison, the percentage rates for the four criteria ranged from $44-91 \%$ for the sepsis only post-intervention group. The timely administration of the appropriate broadspectrum antibiotic ranged from 95 to $100 \%$ for the three levels of sepsis in the postintervention group. Increases in all criteria were realized, and the significant increase in timely antibiotic use was celebrated as a great success.

Prior to intervention of interprofessional real-time coaching, education and a physician order set, when a patient screened positive for sepsis, physicians were reluctant to order the interventions to manage sepsis for several reasons. First, in the early stages, the patient did not look septic leading to the possible belief that treatment was not warranted. Second, this population was young and healthy, resulting in the belief that patients with sepsis were over-treated. A knowledge gap was identified for implementing sepsis bundles for early recognition at this early stage. According to Oud (2007), there were several explanations for this knowledge gap. There is not an abundance of literature on maternal sepsis and/or studies of maternal sepsis had small sample sizes. Of the studies reported, there was a variance in case definition of sepsis and severe sepsis. The adjusted criteria for the SIRS criteria in perinatal patients were not consistent.

To address this knowledge gap, articles were displayed for clinicians to read about the importance of early recognition and management of maternal sepsis. The literature supports the need for hospitals to develop early warning signs for recognition of sepsis as patients can deteriorate rapidly (Surviving Sepsis Campaign, 2012). A study by 
Kramer (2009) reported that the time from first symptom of sepsis to septic shock was less than 24 hours in $39 \%$ of their patients. For women who died of severe sepsis, the time from onset of infection to death was less than 24 hours in $50 \%$ of patients.

In addition, education for clinicians was focused towards providing the rationale for the adjustments of maternal parameters to reflect the physiology of pregnancy. The physician champion presented at meetings attended by physicians, whereas case scenarios were used to help clinicians examine how best practice could be incorporated in the care of the patient. In addition, the physician order set was updated several times to reflect current guidelines or interpretation of the guidelines. The physician champion gave presentations at Grand Rounds to educate physicians on incorporating guidelines of the Surviving Sepsis Campaign for perinatal patients. During work time, the perinatal $\mathrm{RN}$ champions shared literature with physicians and discussed the new perinatal sepsis order set with physicians. These one-to-one interactions allowed for clinicians to discuss concerns, and allowed for an intimate dialogue to problem solve each individual patient situation.

Fears of antibiotic resistance or risk of pulmonary edema were realistic concerns amongst providers. However, pulmonary edema and antibiotic resistance are amenable to treatment, whereas mortality associated with severe sepsis is not. The use of physician champions and pharmacy champions help to dispel these concerns. The effects of these educational interventions lead to a greater understanding of the problem, shared mental model about the evidence based solutions, and ultimately are reflected in an overall increase in bundle compliance indicators in the post-intervention group for patients with sepsis. 


\section{Bundle Compliance Indicators}

Bundle compliance indicators were reviewed for significance in pre and post intervention group. Bundle compliance indicators reaching statistical significance when comparing pre and post-intervention group included draw lactate, repeat lactate and administer broad-spectrum antibiotic(s).

Lactate Level in Sepsis Patients. Bundle compliance for drawing lactate and drawing repeat lactate was significant in sepsis patients in comparison of pre and postintervention group. Lactate level is the most widely used biomarker indicating organ dysfunction (Faix, 2013). The role of lactate as a biomarker was met with resistance due to the possibility that lactate could be falsely elevated in laboring woman due to increased work of the skeletal muscles in labor. Recently, researchers determined normal lactate levels for healthy, non-septic, pregnant women before labor, in the middle of labor (cervical dilation of 6-9 $\mathrm{cm}$ ) and 6 hours postpartum. Ninety-six percent of the lactate levels were below $4 \mathrm{mmol} / \mathrm{L}$ regardless of the stage of labor or postpartum. Therefore, it was believed that lactate level could be used as a reliable biomarker for sepsis in the pregnant patient. Education was provided for clinicians to avoid screening patients for sepsis during the second stage of labor, as the SIRS criteria could not be evaluated accurately. Respiratory rates and heart rates are normally elevated when a laboring woman is pushing during the second stage of labor. In addition, maternal venous lactate increased during the second stage of labor (Nordtrom, Achanna, Naka, and Arulkumaran, 2001). Perinatal healthcare professionals were educated and coached to repeat lactate draw every 6 hours until lactate was less than $2 \mathrm{mmol} / \mathrm{L}$ to make sure lactate was clearing. 
Broad-Spectrum Antibiotic for Sepsis. Administering a broad-spectrum antibiotic within 1 hour of presentation of sepsis reached statistical significance for patients with all groups including sepsis, severe sepsis, and septic shock. Kumar, et al (2006) demonstrated in a study that mortality is decreased with the early administration of a broad-spectrum antibiotic while mortality increased by $7.6 \%$ with each hour of delay. The perinatal sepsis physician order set helped to guide the practitioner in giving the appropriate antibiotic based upon source of infection. Antibiotics safe in pregnant women for common OB infections such as chorioamnionitis and pyelonephritis were included in the physician order set. Antibiotics safe for pregnancy were included in order set for severe sepsis or septic shock. Pharmacy champion(s) presented at Grand Rounds and physician-attended meetings to provide education in choosing the right antibiotic for common OB infections and severe sepsis. Nurses were also educated in the appropriate selection of an antibiotic based upon source of infection leading to a shared mental model for treatment amongst both nurses and physicians.

Intravenous Fluid Bolus and Drawing Blood Cultures. The administration of intravenous fluid bolus and drawing blood cultures did not reach statistical significance when comparing pre and post intervention groups. There was a perceived assumption that the patient was receiving adequate intravenous fluids during labor and prior to epidural anesthesia placement for prevention of hypotension. In addition, there was the perceived risk of pulmonary edema secondary to large intravenous fluid boluses. The reluctance for drawing blood cultures was the perception that the source of the infection was generally known and that it was not necessary in the early stages of sepsis. 


\section{Incidental Findings}

There were two incidental findings noted during this study. The first finding was the lack of inconsistency between physicians regarding the type of antibiotic used. When reviewing charts to determine if an antibiotic was given, it was discovered that there was an inconsistency regarding the type of antibiotic for common pelvic infections, sepsis, and severe sepsis. Statistical information was not measured for the type of antibiotic; however, there was a lack of consistency for using the gold standard regime for treatment of common infections. For example, the gold standard treatment for chorioamnionitis was ampicillin and gentamicin if patient had a vaginal birth (Guinn, 2011). Clindamycin was added to the antibiotic therapy if the patient had a cesarean section. The physician champion and pharmacist provided education for physicians regarding gold standard antibiotic treatment regime at physician-attended meetings with improvement noted over time.

The second incidental finding was the realization that some obstetricians were reluctant to diagnose chorioamnionitis or sepsis when indicators for sepsis and/or chorioamnionitis due to competing priorities. A few of the physicians were focused on their patients' birth experience and overall satisfaction; they were reluctant to separate the mother-baby couplet. At the hospital where this research took place, when a laboring woman was diagnosed with chorioamnionitis, the newborn was admitted to the neonatal intensive care unit (NICU) for 48 hours of antibiotics and labor work in order to rule-out sepsis in the newborn. Consequently, with fewer mothers diagnosed with chorioamnionitis, fewer patients were getting antibiotics or diagnosed with an infection to avoid separation of the mother-baby couplet. Using the hospital policy for definition of 
chorioamnionitis, sepsis, severe sepsis, and septic shock, the accuracy of coding of diagnosis was measured. The patients $(\mathrm{N}=94)$ were coded correctly $64.9 \%$ of the time. Education by physician champion for accurate coding of patients with sepsis and/or common OB infections is ongoing at physician-attended meetings. Contextual factors, such as this variance in diagnosis and treatment, are important to consider when planning future quality improvement activities.

\section{Limitations}

There were several limitations to this study. One, limitation was the effects of previous interventions had on this study. The time period for which the charts were reviewed was not strictly pre- and post-intervention periods of time. The charts were reviewed from April 2014 through January 2015, but the project began in September 2013 with a draft perinatal sepsis order set that was discussed at several physicianattended committee meetings. Some physicians were compelled by the research on maternal sepsis and began using the recommended bundles in management of $\mathrm{OB}$ patients screening positive for sepsis. The informal education began in September 2013. Therefore, this resulted in some bias for the pre-intervention group. It is assumed that greater statistical significance would have been reached if the chart review had been began in September 2013. Therefore, this resulted in some bias for the pre-intervention group. But in light of the pre-intervention data, best practice was definitely not hardwired, and opportunities for improvement were still available.

Another limitation to the chart review process was that post-intervention charts were being reviewed as educational interventions were still in progress. A true postintervention review is planned for 6 months form the last intervention, to see if advances 
have been maintained. Despite both of these limitations to the chart review process, some significant results were still realized. .

The third limitations to the study was the need to interpret some of the chart data. The nurses did not consistently document the administration of the intravenous fluids appropriately. The researcher had to make inferences about fluid boluses according to date and time of physician's order. The fourth limitation was the potential problem of missing some patients who should have been included in the study. The patient entered the study when a rapid response team nurse evaluated the patient. There were most likely patients with sepsis who were treated without a rapid response team nurse evaluating the patient. Therefore, there is a potential for missing patients with sepsis. Patients were not excluded from the study when the nurse performed sepsis screening during the second stage of labor. As a result, this could lead to bias regarding number of patients meeting sepsis, severe sepsis, and septic shock criteria. Future studies could include patient outcomes such as length of stay and ICU admissions.

Lastly, compliance with sepsis bundles was not measured by provider, nor by nurse. The charts that were reviewed were identified by the patient's diagnosis. Therefore, it is impossible to know if all providers and/or all nurses were involved in the care of the patients reviewed in this study. Therefore it is impossible to infer that all of the clinicians have incorporated the sepsis bundles into their practice.

\section{Implications for Nursing Practice}

It is recommended for protocols in the obstetrical service line to include early warning signs for sepsis in perinatal patients. According to California Department of Public Health (2011), early signs of deteriorating condition can be difficult to recognize 
because critical illness is rare in normal pregnancy and childbirth. Developing early warning signs include a clear expectation for observation with predefined criteria for assessment that triggers a response when an abnormality is present (Ivory, 2014). Adjusting the SIRS criteria to account for the physiological changes of pregnancy can assist the clinician with early detection and recognition while triggering a set of interventions for management of maternal sepsis.

Hospitals providing services for perinatal patients should develop standardized procedures and protocols for early recognition and management of maternal sepsis. Using the sepsis bundles to create a clinical protocol that fits the needs of the institution is essential for successful implementation (Surviving Sepsis Campaign, 2012). The purpose of developing protocols for management of maternal sepsis will build a collaborative environment to facilitate change using evidence-based practice. Standardizing care with managing patients with sepsis reduces errors in the delivery of care. The protocols should include all elements of sepsis bundles; however, allow flexibility of the needs of the hospital. Reliability of the bundles has been established to reduce mortality by $25 \%$ in patients' with sepsis

The importance of an implementation plan that used interprofessional education interventions, a standardized protocol, and a multidisciplinary team cannot be overemphasized. It is important to form multidisciplinary teams to develop protocols and guidelines for managing any quality improvement process, including maternal sepsis. Multidisciplinary teams include experts, front-line clinicians, pharmacists, physicians, rapid response team members, emergency room nurses and physicians, and other healthcare professionals who are affected by the protocol. Including a diverse group of 
experts allows for identification of problems with the new patient standard through a multidisciplinary approach

Physician and nurse champions must be identified to mentor staff in signs and symptoms of sepsis, severe sepsis, and septic shock. RN and physician champions can mentor staff in early goal-directed therapy while caring for patients with sepsis.

Including front-line staff in the decision-making and rollout helps to overcome opposition in a manner that allows them to incorporate new ideas into the process and convert possible opponents into supporters (Porter-O-Grady and Malloch, 2011).

\section{Theoretical Framework}

The use of the Iowa Model supported this quality improvement project at an organizational level. The Iowa Model's primary purpose is guiding practitioners in the use of evidence base practice to improve patient outcomes (Titler, M, 2010). Implementing education and a perinatal sepsis physician order set for improving the management of maternal sepsis required teamwork. This project involved multiple departments working together to improve patient safety. Prior to implementing education and physician order set, a small group of stakeholders collaborated together to start researching the guidelines to create the physician order set. This group researched the Surviving Sepsis guidelines and decided that a practice change was warranted. In the planning stage, the Iowa model includes evaluation of case studies, expert opinion, and scientific principles before disseminating information out to healthcare professionals. This step was important as healthcare professionals are exposed to change daily. A wellplanned change project can be the decision factor of whether it is adopted or eliminated. 
Organizational support was crucial for success in implementing this change of practice. Forming a multidisciplinary team including nurses, rapid response team nurses, physician champions, pharmacists, clinical nurse specialists, and leadership team members helped multiple departments decide how to roll out this change project effectively. The team collaborated periodically to refine the process through evaluation of the change project. This evaluation process was an important part of the success of the project. Team members collaborated to identify knowledge gaps, improvements in the process, and problematic areas that required revision. It was through refining the process, that healthcare professionals adopted this change project. The use of physician champions and nurse champions helped to monitor and analyze the process of managing patients with sepsis. In addition, the use of champions helped to mentor healthcare professionals during change process while managing septic patients.

Organizational context was important to consider. The hospital where the project took place was in the midst of preparing to move into a new building and to implement an electronic medical record, all on the same day. In addition, the department had recently finished an 18 month quality improvement project focused on practice changes for preeclampsia. Resources were minimal, and the leadership team was somewhat distracted by other commitments. The success of this project is a testament to the strength of bedside nurses, in collaboration with other key stakeholders, to engage, energize and achieve outcomes in the midst of organizational stress. In addition, bedside nurses have the unique understanding of clinicians' concerns, and have their ear when real-time orders and decisions are being made. The use of the Iowa model (Titler, 2010) 
has provided a structure to implement quality improvement strategies involving multiple departments in a tertiary care hospital.

\section{Summary}

The purpose of this doctoral quality improvement project was to evaluate staff compliance with early goal directed therapy before and following the implementation of standardized physician order and interprofessional education for nurses and physicians in the perinatal setting. The systemic inflammatory response criteria were adjusted for consideration of the physiological effects of pregnancy to accurately screen for sepsis. When determining the effects of interprofessional education and a physician order set, statistical significance was achieved for drawing lactate, administering a broad spectrum antibiotic, and drawing repeat lactate in patients with sepsis. In addition, statistical significance was achieved for administering a broad-spectrum antibiotic for patients with severe sepsis and septic shock, with $100 \%$ compliance achieved for both these diagnoses. The value of using a multi-disciplinary team and a group of primary care nurses to implement interprofessional education and institute standardized physician order set has been established. Taking into consideration contextual factors specific to this hospital, this project made significant progress in meeting the recommendation for early recognition and management of maternal sepsis by the Surviving Sepsis Campaign (2012).

Further research is needed in looking specifically at physiologic criteria and laboratory values for screening the perinatal patient at risk for sepsis. In addition, there needs to be more research done to determine normal lactate parameters for pregnancy, early labor, active labor, second stage of labor, and the immediate postpartum. Lactate is 
a valuable biomarker for inadequate tissue perfusion and its value in the obstetrical patients' needs to be validated. 
REFERENCES 


\section{REFERENCES}

Acosta, C. and Knight, M. (2013). Sepsis and maternal mortality. Maternal-Fetal Medicine, 25(2), 109-116.

Acosta, C. D., Knight, M., Lee, H. C., Kurinczuk, J. J., Gould, J. B., \& Lyndon, A. (2013). The Continuum of Maternal Sepsis Severity: Incidence and Risk Factors in a Population-Based Cohort Study. Plos ONE, 8(7), 1-8. doi:10.1371/journal.pone.0067175.

Acosta, C. Kurinczuk, J. Lucas, Nuala, D.L., Tuffnell, D. Sellers, S. Knight, M. (2014). Severe maternal sepsis in the U.K., 2011-2012: A national casecontrol study. Plos Medicine, 11(7), 1-15.

Bamfo, J. (2013). Managing the risks of sepsis in pregnancy. Best Practice \& Research: Clinical Obstetrics \& Gynaecology, 27(4), 583595. doi:10.1016/j.bpobgyn.2013.04.003.

Barton, J., and Sibai, B. (2012). Severe sepsis and septic shock in pregnancy. Obstetrics \& Gynecology, 120(3), 689-706. doi:10.1097/AOG.0b013e318263a52d

Bauer, M., Bateman, B., Bauer, S., Shanks, A., and Mhyre, J. (2013). Maternal sepsis mortality and morbidity during hospitalization for delivery: Temporal trends and independent associations for severe sepsis. Society for Obstetric Anesthesia and Perinatology, 117(4), 943-950. 
Black, L.P., Hinson, L. and Duff, P. (2012). Limited course of antibiotic treatment for chorioamnionitis. Obstetrics and Gynecology, 119(6), 1102-1105. California Department of Health. (2011). The California pregnancy-associated mortality review. Report from 2002 and 2003 maternal death reviews. Sacramento, CA: Author. Retrieved from http://www.cdph.ca.gov/data/statistics/Documents/gMO-CA-PAMRMaternalDeathReview-2002-03.pdf.

Centre for Maternal and Child Enquiries. (2011). saving mothers' lives: Reviewing maternal deaths to make motherhood safer: 2006-2008, BJOG: An International Journal of Obstetrics and Gynaecology, 18(Suppl. 1), 1205.

Ciliska, D. DiCenso, A., Melnyk, B.M., Finout-Overhold, E. Stetler, C., Cullen, L., Larrabee, J., Schultz, A. Rycroft-Malone, J., Newhours, R., and Dang, D. In Melnyk, B. and Fineout-Overholt, E. (2011). Evidence-based practice in nursing \& healthcare $\left(2^{\text {nd }} E d\right.$.). Philadelphia, PA: Lippincott Williams \& Wilkins.

Dellinger, R.P, Levy, M.M., Carlet, J.M., Bion, J., Parker, M.M., Jaeschke, R., Reinhart, K., Angus, D.C., Brun-Buisson, D., Beale, R., Calandra, T., Dhainaut, J.F., Gerlach, H., Harvey, M., Marini, J.J., Marshall, J., Ranieri, M., Ramsay, G., Sevransky, J., Thompson, B.T., Townsend, S., Vender, J.S., Zimmerman, J.L., Vincent, J.L. (2012). Surviving sepsis 
campaign: International guidelines for management of severe sepsis and septic shock. Critical Care Medicine, 36, 97-327.

Faix, J. (2013). Biomarkers of sepsis. Critical Review Clinical Laboratory Science, 50(1), 24-36. Doi: 10.3109/10408363.2013.764490.

Gonen, R., Korobochka, R., Degani, S., and Gaitini, L. (2000). Association between epidural analgesia and intrapartum fever. American Journal of Perinatology, 17(3), 127-1008.

Guinn, D., Abel, D. and Tomlinson, M. (2007). Early goal directed therapy for sepsis during pregnancy. Obstetrics and Gynecology Clinics of North America, 34, 59-479.

Guinn, D. (2011). Maternal sepsis 2010: Early recognition and aggressive treatment with early goal-directed therapy could improve maternal outcomes. Current Women's Health Reviews, 7(2), 164-176.

Honiden, S., Abdel-Razeq, S. and Siegel, M., 2011. The management of the critically ill obstetric patient. Journal of Intensive Care Medicine, 28(93), 93-105.

Ivory, C.H. (2014). Standardizing the words nurses use to document elements of perinatal failure to rescue. Journal of Obstetric, Gynecologic, \& Neonatal Nursing, 43(1), 13-24.

Joseph, J. Sinha, A., Paech, M. and Walters, B. (2009). Sepsis in pregnancy and early goal-directed therapy. Obstetrics Medicine, 2, 93-99. 
Kirchner, J. E., Parker, L. E., Bonner, L. M., Fickel, J. J., Yano, E. M., \& Ritchie, M. J. (2012). Roles of managers, frontline staff and local champions, in implementing quality improvement: stakeholders' perspectives. Journal of Evaluation in Clinical Practice, 18(1), 63-69. doi:10.1111/j.13652753.2010.01518.x

Kramer, H. C., Schutte, J. M., Zwart, J. J., Schuitemaker, N. E., Steegers, E. P., \& Van Roosemalin, J. (2009). Maternal mortality and severe morbidity from sepsis in the Netherlands. Acta Obstetricia Et Gynecologica Scandinavica, 88(6), 647-653. Doi: 10.1080/00016340902926734

Kumar, A, Roberts, D. Wood, K.E., et al. (2006). Duration of hypotension before initiation of effective antimicrobial therapy is the critical determinant of survival in human septic shock. Critical care Medicine, 34, 589-596.

Lapinsky, S. (2013). Obstetric infections. Critical Care Clinics, 29,509-520.

Martin, G.S., Mannino, D.M., Eaton, S, Moss, M. (2003). The epidemiology of sepsis in the United States from 1970 through 2000. New England Journal of Medicine, 348, 1546-1554.

Melynk, G. \& Fineout-Overholt, E. (2011). Evidence-based practice in nursing \& healthcare (2 $2^{\text {nd }}$ Ed.). Philadelphia, PA: Lippincott Williams \& Wilkins. Morgan, J. and Roberts, S. (2012). Maternal sepsis. Obstetrics and Gynecology Clinics of North America, (40), 69-87. 
Nordstrom, L., Achanna, S. Naka, K. Arulkumaran, S. (2001). Fetal and maternal lactate increase during active second stage of labour. British Journal of Obstetrics and Gynaecology, 108, 263-268.

Nguyen, H.M., Schiavoni, A., Scott, K.D., and Tanios, M.A. (2012). Implementation of sepsis management guideline in a community-based teaching hospital-can education be potentially beneficial for septic patients? International Journal of Clinical Practice, 66(7), 705-710.

Oud, L. (2014). Pregnancy-associated severe sepsis: Contemporary state and future challenges. Infectious Disease Therapy, 3, 175-189.

Porter-O'Grady, T. and Malloch, K. (2011). Quantum Leadership: Advancing Innovation: Transforming Health Care ( $3^{\text {rd }}$ Ed.). Sudbury, MA: Jones \& Bartlett.

Rivers, E., Nguyen, B., Havstad, S. Ressler, J., Muzzin, A., Knoblich, B., Peterson, E., Tomlanovich, M. (2001). Early goal-directed therapy in the treatment of severe sepsis and septic shock. The New England Journal of Medicine, 345(19), 1368-1377.

Royal College of Obstetricians and Gynaecologists (2012). Bacterial sepsis in pregnancy: Green-top guideline No. 64a. Retrieved from http://www.rcog.org.uk/files/rcog-corp/25.4.12GTG64a.pdf

Sanghavi, M. and Rutherford, J.D. (2014). Cardiovascular physiology of pregnancy. Circulation, 130: 1003-1008. 
Shaw, E., Howard, J., West, D., Crabtree, B., Nease, D., Tutt, B., \& Nutting, P. (2012). The role of the champion in primary care change efforts: From the state networks of Colorado ambulatory practices and partners (SNOCAP). The Journal of the American Board of Family Medicine, 25(5), 676-685.

Snyder, D., Barton, J. Habli, M. and Sabai, B. (2013). Severe sepsis and septic shock in pregnancy: Indications for delivery and maternal and perinatal outcomes. The Journal of Maternal-Fetal and Neonatal Medicine, 26(5), 503-506.

Stephens-Hennessy, B.M. (2012). Sepsis screening in the perinatal patient. Journal of Obstetric, Gynecologic, and Neonatal Nursing, 14, S24. Doi:10.1111/j.1552-6909.2012.01359_29.x.

Sung, E., George, J. and Porter, M. (2011). Sepsis in pregnancy. Fetal and Maternal Medicine Review, 22(4), 287-305.

Surviving Sepsis Campaign (2012). Retrieved from http://www.survivingsepsis.org/Guidelines/Pages/default.aspx Taylor-Piliae (1999). Utilization of the Iowa Model in establishing evidencebased nursing practice. Intensive and Critical Care Nursing, 15, 357-362.

Titler, M.G. (2010), Iowa model of evidence-based practice. In Malone, J. \& Bucknall, T. Models and frameworks for implementing evidence-based practice. Malden, MA: Sigma Theta Tau International Honor Society of Nursing. 
Waterstone, M., Bewley, S. and Wolfe, C. (2001). Incidence and predictors of severe obstetric morbidity: case-control study. British Medical Journal, 322: 1089-1091.

Yeomans, E. and Gilstrap, L (2005). Physiologic changes in pregnancy and their impact on critical care. Critical Care Medicine, 33(10), S256-S258. 
APPENDICES 


\section{APPENDIX A: IOWA MODEL}




\section{The lowa Model of Evidence-Based Practice to Promote Quality Care}

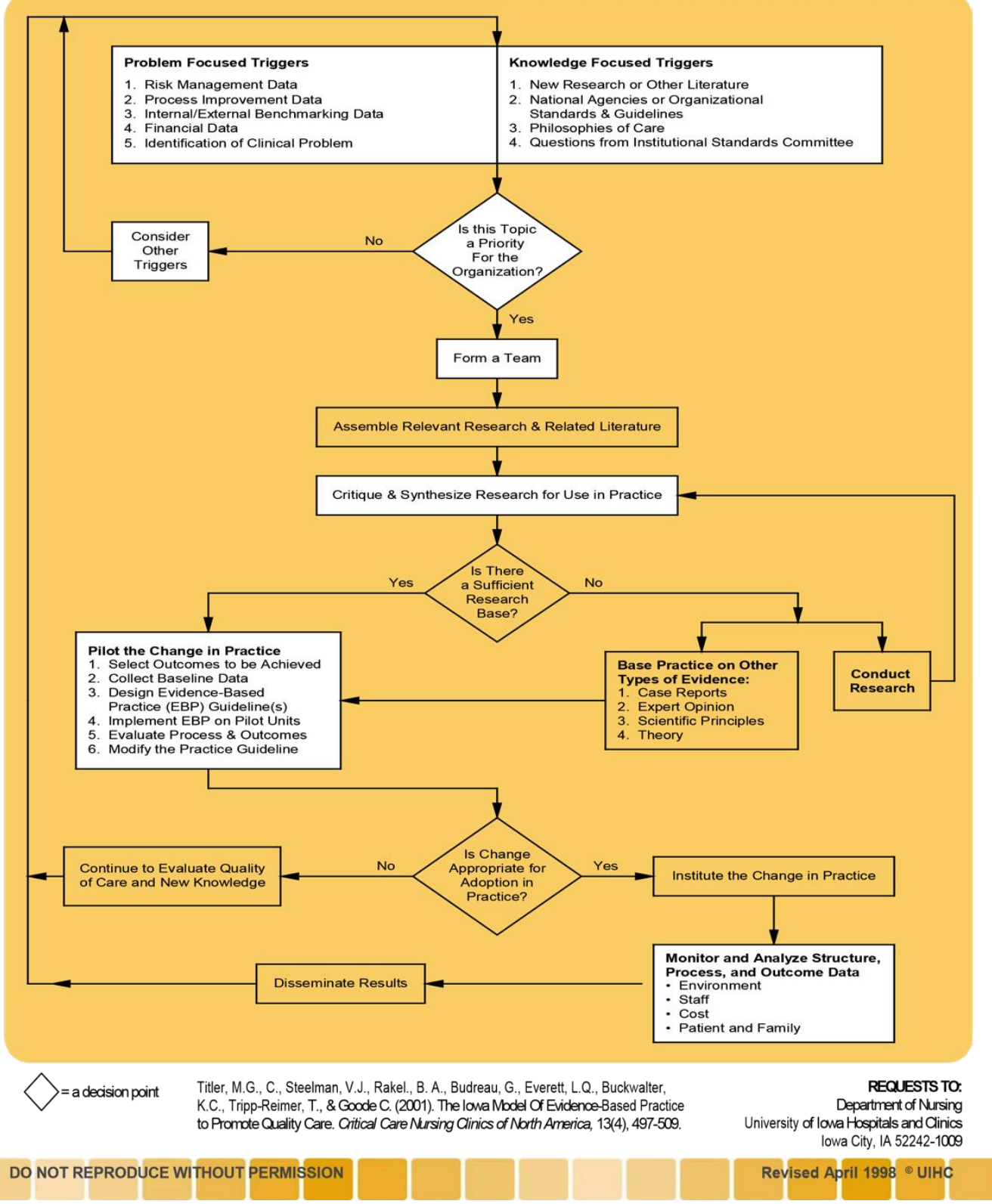

Used/Reprinted with permission from the University of Iowa Hospitals and Clinics and Marita G. Titler, PhD, RN, FAAN. Copyright 1998. For permission to use or reproduce the model, please contact the University of Iowa Hospitals and Clinics at (319)384-9098 
APPENDIX B: STANDARDIZED PHYSICIAN ORDER SET 
Date:

Time:

Allergies:

Diagnosis:

If none selected Notify MD immediately. Ensure blood cultures are obtained prior to antibiotic initiation. SYSTEMIC INFLAMMATORY RESPONSE CRITERIA

\section{If $\mathbf{2}$ or more of following SIRS criteria are present with documented or suspected infection}

- Temp greater than $38.3^{\circ} \mathrm{C}\left(101^{\circ} \mathrm{F}\right)$ or $<36^{\circ} \mathrm{C}\left(86.8^{\circ} \mathrm{F}\right)$

- HR greater than $100 \mathrm{bpm}$

- RR greater than 20 breaths/minute

- WBC greater than 20,000 (in labor), greater than 12,000 (not in labor), $<4,000$ OR greater than $10 \%$ immature neutrophils

- New mental status change

- Blood glucose $\geq 140 \mathrm{mg} / \mathrm{dl}$ in absence of diabetes

\begin{tabular}{ll}
\hline & INTERVENTIONS FOR SEPSIS \\
$\square$ Initiate peripheral IV _ _ \#16 gauge __ \#18 gauge using $1 \%$ lidocaine per protocol. \\
$\square \mathrm{IV} \_$at
\end{tabular}

$\square$ Initiate Rapid Response Team

$\square$ Vital Signs (BP, P, R) every 30 min X 4, every 1 hour X 4, then every shift

$\square$ Temperature every 2 hours

$\checkmark \mathrm{O} 2$ per oxygen therapy protocol - keep 02 saturation greater than or equal to $92 \%$

$\checkmark$ Initiate antibiotics within 60 minutes of "identified sepsis" considering source of infection

$\square$ Lactate STAT and every 6 hours $\times 4$

LABS AND RADIOLOGY STUDIES

$\square \mathrm{CBC}$

$\square$ Blood Cultures $\times 2$ (prior to administration of antibiotics)

$\square$ Comprehensive Metabolic Panel

$\square$ Protime $\square$ Partial Thromboplastin Time

UA and culture if indicated

Arterial Blood Gas (for respiratory symptoms)

$\square$ Chest X-ray (PA \& LAT) Indication:

Serum Creatinine

\section{ACUTE ORGAN DYSFUNCTION CRITERIA}

If patients have one of these criteria $\rightarrow$ Acute Organ Dysfunction $\rightarrow$ SEVERE SEPSIS

- SBP $<90 \mathrm{mmHG}$ or MAP $<85 \mathrm{mmHG}$ and HR $\geq 100$

- SBP decrease greater than $40 \mathrm{mmHG}$ from baseline and HR $\geq 100$

- New (or increased) oxygen requirement to maintain SP02 greater than $90 \%$

- Bilirubin greater than $2 \mathrm{mg} / \mathrm{dl}$

- Platelet count $<100,000$

- Coagulopathy (INR greater than 1.5 or aPTT greater than 80 secs)

- Lactate $\geq 2$ mmoll

$\checkmark$ IV fluid bolus $0.9 \%$ NS now $(30 \mathrm{~mL} / \mathrm{kg}$ )

$\square$ Strict Intake and Output. Notify MD urine output less than $0.5 \mathrm{~mL} / \mathrm{kg} / \mathrm{hr}$

$\square$ Initiate Rapid Response Team

If Systolic BP $<90$ or MAP $<85$ after initial fluid bolus, or lactate $>3.9$, NOTIFY MD

$\square$ Notify MD for signs of acute organ dysfunction criteria FOR SEPTIC SHOCK (Systolic $<90$, MAP $<65$ or Lactate greater than $3.9 \mathrm{mmol} /$ )

$\checkmark$ IV fluid bolus $0.9 \%$ NS now ( $30 \mathrm{~mL} / \mathrm{kg}$ )

$\checkmark$ Notify MD for transfer to ICU

$\checkmark$ Notify MD for systolic BP less than 90, MAP less than 65 or lactate greater than 3.9

$\square$ Place foley catheter with urometer

$\square$ Strict Intake and Output. Notify MD urine output less than $0.5 \mathrm{~mL} / \mathrm{kg} / \mathrm{hr}$

$\checkmark$ Initiate Rapid Response Team (if not already present)

$\square$ Initiate broad spectrum antibiotics within 60 minutes of "identified sepsis"

Sutter Health

Sacramento Sierra

Region

DSAFH

$\square$ SAH

$\square \mathrm{SDH}$

DSMCS

$\square$ SRMC

Perinatal Sepsis Orders

Authorization for therapeutic substitution is given unless checked here

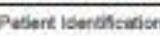


* Infuse first antibiotic within the $1^{\text {tt }}$ hour and ensure both antibiotics are infused within 3 hours.

* If no antibiotics selected, notify MD immediately. Ensure blood cultures are obtained prior to antibiotic initiation.

\section{ANTIBIOTICS FOR SEVERE SEPSIS / SEPTIC SHOCK}

Unknown etiology and/or currently on any above antibiotics

Discontinue all current antibiotic(s)

$\square$ Vancomycin $20 \mathrm{mg} / \mathrm{kg}$ (up to $2 \mathrm{gm}$ ) IV now and continue per pharmacy dosing:

AND one of the antibiotics below:

Zosyn $4.5 \mathrm{gm}$ IV now and continue per pharmacy dosing (give first):

OR

$\square$ For Non Significant or Unknown Penicillin Allergy:

Cefepime $2 \mathrm{gm}$ IV now and continue per pharmacy dosing

$\square$ For Significant Penicillin Allergy (anaphylaxis, angioedema, respiratory distress, urticaria):

Aztreonam $2 \mathrm{gm}$ IV now and continue per pharmacy dosing

OR

Gentamicin $5 \mathrm{mg} / \mathrm{kg}$ IV now and continue per pharmacy dosing

Additional orders:

Date: Time:

Physician Signature:

Authorization for therapeutic substitution is given unless checked here

Sutter Health

Sacramento Sierra

Region

QSAFH $\square$ SAH $\square$ SDH $\square$ SMCS $\square$ SRMC QSSMC

Perinatal Sepsis Orders Page 2 of 2

OADERS 
APPENDIX C: OBSTETRICAL SEPSIS PATHWAY 


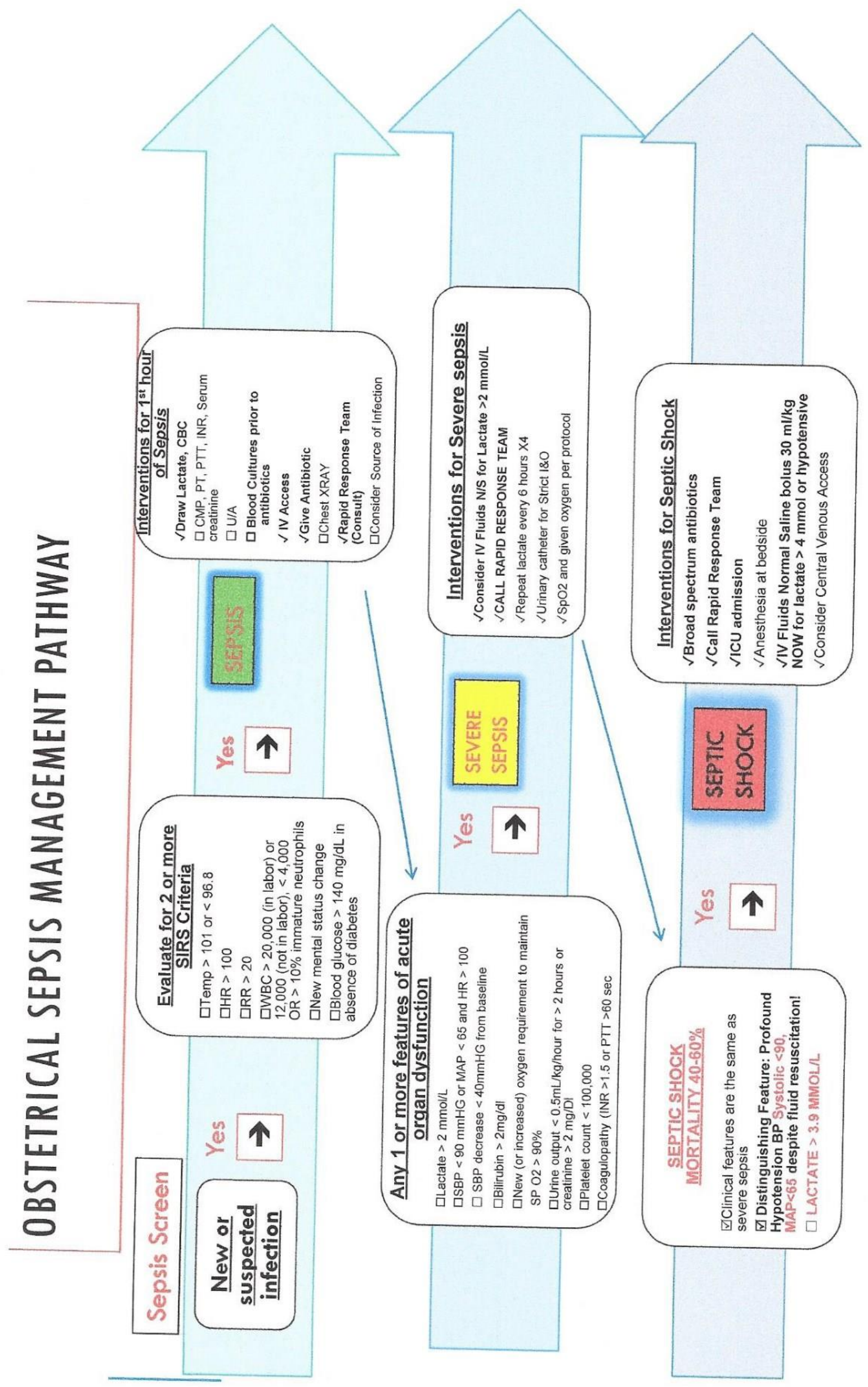


APPENDIX D: SEPSIS DATA COLLECTION TOOL 


\begin{tabular}{|c|c|c|c|c|c|c|c|c|c|c|c|c|c|c|c|c|}
\hline ID & $M D$ & $\mathrm{RN}$ & Unit & $\begin{array}{l}\text { Sepsis } \\
\text { Y/N }\end{array}$ & $\begin{array}{l}\text { Severe } \\
\text { Sepsis } \\
Y / N\end{array}$ & $\begin{array}{l}\text { Diagnosis } \\
\text { (List) }\end{array}$ & $\begin{array}{l}\text { Lactate } \\
\text { yes/no }\end{array}$ & $\begin{array}{l}\text { Lactate } \\
\text { level }\end{array}$ & $\begin{array}{l}\text { Blood } \\
\text { Culture } \\
\text { Before } \\
\text { ATB } \\
\text { Y/N } \\
\end{array}$ & $\begin{array}{l}\text { ATB 3 hr } \\
\text { Outpt } \\
\text { (Y/N) }\end{array}$ & $\begin{array}{l}\text { ATB } 1 \mathrm{hr} \\
\text { inpt } \\
\text { (Y/N) }\end{array}$ & $\begin{array}{l}\text { Fluid Bolus } \\
30 \mathrm{ml} / \mathrm{kg} \\
(\mathrm{Y} / \mathrm{N})\end{array}$ & $\begin{array}{l}\text { All yes } \\
\text { within } 3 \\
\text { hours } \\
\mathrm{Y} / \mathrm{N}\end{array}$ & $\begin{array}{l}\text { Rep } \\
\text { lactate } \\
\text { in } 6 \\
\text { hours } \\
\mathrm{Y} / \mathrm{N} \\
\end{array}$ & $\begin{array}{l}\text { ICU } \\
\text { Admi } \\
t \\
Y / N\end{array}$ & $\begin{array}{l}\text { Code } \\
\mathrm{Y} / \mathrm{N}\end{array}$ \\
\hline & & & & & & & & & & & & & & & & \\
\hline & & & & & & & & & & & & & & & & \\
\hline & & & & & & & & & & & & & & & & \\
\hline & & & & & & & & & & & & & & & & \\
\hline & & & & & & & & & & & & & & & & \\
\hline & & & & & & & & & & & & & & & & \\
\hline & & & & & & & & & & & & & & & & \\
\hline & & & & & & & & & & & & & & & & \\
\hline & & & & & & & & & & & & & & & & \\
\hline & & & & & & & & & & & & & & & & \\
\hline
\end{tabular}


APPENDIX E: SEPSIS SCREEN 


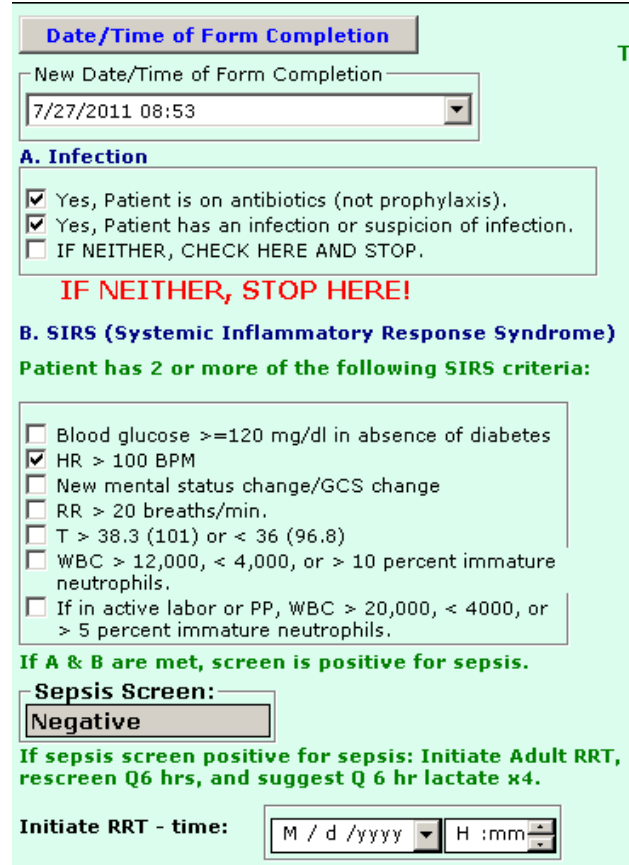

SEPSIS SCREEN

To be completed every shift and PRN.

C. ACUTE Organ Dysfunction

Patient meets one or more of the following criteria:

I SBP $<90 \mathrm{mmHG}$ or MAP $<65 \mathrm{mmHG}$ with $\mathrm{HR}>=100$.

I SBP decreased $>40 \mathrm{mmHG}$ from baseline.

$\Gamma$ New (or increased) oxygen requirement to maintain $\mathrm{SpO} 2>90$.

C Urine Output $<=20 \mathrm{ml} / \mathrm{hr}$, or Creatinine $>=2 \mathrm{mg} / \mathrm{dl}$.

C Bilirubin $>2 \mathrm{mg} / \mathrm{dl}$.

I Platelet count $<100,000$.

Coagulopathy (INR $>1.5$ or aPTT $>60$ secs).

Lactate $>=2 \mathrm{mmol} / \mathrm{L}$

If all criteria met for A, B \& C, screen is positive for SEVERE sepsis.

New Severe Sepsis Screen

Negative

$\lceil$ New positive screen for severe sepsis/ RRT called sepsis alert.

MD notified:

$\lceil$ Transferred to ICU $\square$ Transferred to Higher Level of Care. 